\title{
Multiplicity of ground states for the scalar curvature equation
}

\author{
Francesca Dalbono ${ }^{1} \cdot$ Matteo Franca $^{2}$ (D) Andrea Sfecci $^{3}$
}

Received: 6 January 2019 / Accepted: 5 June 2019 / Published online: 13 June 2019

(c) Fondazione Annali di Matematica Pura ed Applicata and Springer-Verlag GmbH Germany, part of Springer Nature 2019

\section{Abstract}

We study existence and multiplicity of radial ground states for the scalar curvature equation

$$
\Delta u+K(|x|) u^{\frac{n+2}{n-2}}=0, \quad x \in \mathbb{R}^{n}, \quad n>2,
$$

when the function $K: \mathbb{R}^{+} \rightarrow \mathbb{R}^{+}$is bounded above and below by two positive constants, i.e. $0<\underline{K} \leq K(r) \leq \bar{K}$ for every $r>0$, it is decreasing in $(0,1)$ and increasing in $(1,+\infty)$. Chen and Lin (Commun Partial Differ Equ 24:785-799, 1999) had shown the existence of a large number of bubble tower solutions if $K$ is a sufficiently small perturbation of a positive constant. Our main purpose is to improve such a result by considering a non-perturbative situation: we are able to prove multiplicity assuming that the ratio $\bar{K} / \underline{K}$ is smaller than some computable values.

Keywords Scalar curvature equation - Ground states · Fowler transformation · Invariant manifold $\cdot$ Shooting method $\cdot$ Bubble tower solutions $\cdot$ Phase plane analysis $\cdot$ Multiplicity results

Mathematics Subject Classification $35 \mathrm{~J} 61 \cdot 37 \mathrm{D} 10 \cdot 34 \mathrm{C} 37$

F. Dalbono: Partially supported by the GNAMPA project "Dinamiche non autonome, analisi reale $e$ applicazioni". M. Franca: Partially supported by the GNAMPA project "Sistemi dinamici, metodi topologici e applicazioni all'analisi nonlineare". A. Sfecci: Partially supported by the GNAMPA project "Problemi differenziali con peso indefinito: tra metodi topologici e aspetti dinamici".

Matteo Franca

franca@dipmat.univpm.it

Francesca Dalbono

francesca.dalbono@unipa.it

Andrea Sfecci

asfecci@units.it

1 Dipartimento di Matematica e Informatica, Università degli Studi di Palermo, Via Archirafi 34, 90123 Palermo, Italy

2 Dipartimento di Ingegneria Industriale e Scienze Matematiche, Università Politecnica delle Marche, Via Brecce Bianche 1, 60131 Ancona, Italy

3 Dipartimento di Matematica e Geoscienze, Università degli Studi di Trieste, Via Valerio 12/1, 34127 Trieste, Italy 


\section{Introduction}

This paper is devoted to the study of existence and multiplicity of positive solutions for the scalar curvature equation

$$
\Delta u+K(|x|) u^{\frac{n+2}{n-2}}=0,
$$

where $x \in \mathbb{R}^{n}, n>2$, and $K$ is a reciprocally symmetric, bounded, positive, continuous function, $C^{1}$ for $r=|x|>0$. The main purpose is to ensure the multiplicity of positive entire solutions which decay at infinity like $|x|^{2-n}$ (i.e. fast decay solutions), when

$$
K \text { decreases in }(0,1) \text { and } K \text { increases in }(1, \infty) .
$$

According to [3, Theorem 1], [5, Theorem 2], we know that assumption (1.2) guarantees that each solution of (1.1) is radially symmetric about the origin.

Therefore, it is not restrictive to concentrate on radial solutions of (1.1), by considering the equivalent singular O.D.E.

$$
\left(u^{\prime} r^{n-1}\right)^{\prime}+K(r) r^{n-1} u^{\frac{n+2}{n-2}}=0, \quad r \in(0, \infty),
$$

where "' " denotes the differentiation with respect to $r=|x|$, and, with a slight abuse of notation, $u(r)=u(x)$.

The solutions of (1.3) can be classified according to their asymptotic behaviour at zero and at infinity. More precisely, a solution $u(r)$ of (1.3) is called regular if $u(0)=d$ and $u^{\prime}(0)=0$ and it will be denoted by $u(r ; d)$, and singular if $\lim _{r \rightarrow 0} u(r)= \pm \infty$; similarly, $u(r)$ is a fast decay solution if $\lim _{r \rightarrow \infty} u(r) r^{n-2}=c$, and a slow decay solution if $\lim _{r \rightarrow \infty} u(r) r^{n-2}= \pm \infty$.

Moreover, we say that $u(r)$ is a ground state (G.S.) if it is a positive regular solution of (1.3) such that $\lim _{r \rightarrow \infty} u(r)=0$; we say that $u(r)$ is a singular ground state (S.G.S.) if it is a positive singular solution of (1.3), which is defined for any $r>0$ and satisfies $\lim _{r \rightarrow \infty} u(r)=0$. It is easy to show that G.S. and S.G.S. are decreasing, see Remark 2.5.

Equation (1.1) and its generalizations have attracted the attention of several different authors giving rise to a huge literature on the topic, both for its intrinsic mathematical interest and for its relevance in application. In fact, Eq. (1.1) is known as scalar curvature equation since the existence of G.S. with f.d. is equivalent to the existence of a metric in $\mathbb{R}^{n}$ conformally equivalent to the Euclidean metric and which has scalar curvature $K$, see, for example, $[6,9,14]$ for more details.

Furthermore, Eq. (1.1) finds application in astrophysics for particular type of $K$ : a significant example is given by the Matukuma equation where $u$ represents the gravitational potential in a globular cluster (cf., among others, $[1,11,40]$ ). Finally, it can be used to study the stationary states for a nonlinear Schrodinger equation in quantum mechanic and for a reaction diffusion equation in chemistry. In most of the applications, positivity is crucial and the fast decay is needed to deal with physically relevant solutions.

In the 1980s, it was realized that the Pohozaev function $P(r)=\int_{0}^{r} K^{\prime}(s) s^{n-1} \mathrm{~d} s$ plays a key role in determining the structure of positive solutions of (1.3). No G.S. with fast decay can exist if $P(r)$ has constant sign, so, in particular, when $K$ is monotone (but non-constant), see [14,26,28,32]; while there are many different existence results when $P(r)$ changes sign. The situation is simpler if $P(r)$ is positive for $r$ small and negative for $r$ large, in particular when $K(r)$ has a maximum, see, for example, [6,7,11,28,29,38,39]. More in detail, the existence of a radial G.S. with fast decay was proved requiring that

$$
K(r)=A_{0} r^{\delta_{0}}+A_{1} r^{\delta_{1}} \text { as } r \rightarrow 0, \quad K(r)=B_{0} r^{-\eta_{0}}+B_{1} r^{-\eta_{1}} \text { as } r \rightarrow \infty,
$$


where $A_{0}, B_{0}, \delta_{0}, \eta_{0}$ are positive constants, $\delta_{1}>\delta_{0}, \eta_{1}>\eta_{0}$, see $[11,28,38,39]$. Results are also available when $\delta_{0}=\eta_{0}=0$ and $A_{i}, B_{i}, \delta_{1}, \eta_{1}$ are positive, see, for example, $[6,7,29,39]$. We emphasize that under assumption (1.4) a complete classification of both regular $[27,37,40]$ and singular solutions [13] is available, and the uniqueness of radial G.S. with fast decay is guaranteed whenever the unique critical point of $K$ is a maximum. More precisely, if $K(r)$ satisfies (1.4) with $A_{i}, B_{i}, \delta_{i}, \eta_{i} \geq 0$, and $r K^{\prime} / K$ is decreasing, then there is a unique $d^{*}>0$ such that $u(r ; d)$ is a G.S. with slow decay if $0<d<d^{*}$, it is a G.S. with fast decay if $d=d^{*}$ and it has a positive zero if $d>d^{*}$, see in particular $[27,37,40]$ concerning the uniqueness of $d^{*}$. Furthermore, there are uncountably many radial S.G.S. with slow decay and uncountably many radial S.G.S. with fast decay. Nowadays, also nodal solutions are classified [13,31], and many results have been extended to a $p$-Laplace context $[17,18,24]$. We remark that the presence of a local maximum allows existence and multiplicity of non-radial positive solutions of (1.1), cf. [33] and [35], respectively.

However, there is a striking difference in the structure of radial positive solutions between the case in which $K$ admits a unique maximum and the case in which it admits a unique minimum. As observed above, in the former (and easier) case we could have a unique G.S. with fast decay of (1.3), and a complete classification of the solutions. In the latter (and more complicated) situation, we range from a large number of G.S. with fast decay to nonexistence results, see, for example, [6] for non-existence, [6,7] for existence, and [1,20,21] for both multiplicity and non-existence results in the case where $K$ is an unbounded function satisfying $K(r) \sim r^{\delta}$ as $r \rightarrow 0$ and $K(r) \sim r^{\eta}$ as $r \rightarrow+\infty$, with $\delta<0<\eta$. Partial structure results have been achieved, cf. [20].

Similarly, for $K(r)$ varying between two positive constants the situation is delicate and quite complicated: non-existence results as well as existence of non-radial solutions for (1.1) can be achieved, cf. [7, Theorem 0.4] and [4], respectively.

In the 1990s, it was noticed that multiplicity results could be produced requiring several sign changes in the Pohozaev function, namely asking the function $K$ to have many critical points, under the additional assumption that $K$ is either a regular or a singular perturbation of a constant, i.e.

$$
\begin{aligned}
& K(|x|)=1+\varepsilon k(|x|), \quad 0<k(|x|)<1, \\
& K(|x|)=k\left(|x|^{\varepsilon}\right), \quad k(|x|) \text { bounded, }
\end{aligned}
$$

see $[2,25]$. Some further results in this direction are contained in $[8,36]$.

Chen and Lin in [9] noticed that if $K(|x|)$ has a critical point, but it is a minimum, uniqueness of the G.S. might be lost. They considered $K(|x|)$ as in (1.5) and assume the following

(Ko $K(r)=K\left(\frac{1}{r}\right)$ for any $0<r \leq 1$;

(K1) $K^{\prime}(r) \leq 0$ for any $r \in(0,1)$, but $K^{\prime}(r) \not \equiv 0$;

$\left(\mathbf{K}_{2}\right)$

$$
\begin{gathered}
K(r)=K(0)-A r^{l}+h(r) \quad \text { where } \\
A>0, \quad 0<l<\frac{n-2}{2}, \quad \lim _{r \rightarrow 0}|h(r)| r^{-l}+\left|h^{\prime}(r)\right| r^{-l+1}=0 .
\end{gathered}
$$

Theorem A [9, Theorem 1.1] Assume that $K$ satisfies (1.5) and $\left(\mathbf{K}_{\mathbf{0}}\right)-\left(\mathbf{K}_{\mathbf{1}}\right)-\left(\mathbf{K}_{\mathbf{2}}\right)$, then for any $\ell \in \mathbb{N}$ there exists a $\varepsilon_{\ell}>0$ such that for every $\varepsilon \in\left(0, \varepsilon_{\ell}\right)$ Eq. (1.3) admits at least $\ell$ G.S. with fast decay $u_{1}, \ldots, u_{\ell}$, where the function $u_{j}(r) r^{\frac{n-2}{2}}$ has $j$ local maxima and $(j-1)$ local minima. 
Lin and Liu [30] obtained a similar result, again in a perturbative setting for $K$ of the form (1.5), removing the technical symmetry assumption $\left(\mathbf{K}_{\mathbf{0}}\right)$, but requiring condition $\left(\mathbf{K}_{\mathbf{2}}\right)$ with a more restrictive smallness assumption on the parameter $l$. The same conclusion as in Theorem A was also obtained in [15] for $K$ of the form (1.6), just requiring that $K$ has a (possibly degenerate) positive minimum. As far as we are aware, $[9,15,30]$ are the only papers obtaining multiplicity of G.S. with fast decay for (1.3) with a unique positive minimum of $K$.

The main purpose of this article is to extend the perturbative result of [9] to a nonperturbative situation. To this aim, we give a new argument to reprove Theorem A which furnishes a precise estimate on how small $\varepsilon_{\ell}$ should be, and we show that $\varepsilon_{\ell}$ need not be too small.

Theorem 1.1 All the constants $\varepsilon_{\ell}$ in Theorem A can be explicitly computed. In particular, we can find the following approximations from below:

\begin{tabular}{c|c|c|c|c|c|c|c|c}
$n$ & $\varepsilon_{1}$ & $\varepsilon_{2}$ & $\varepsilon_{3}$ & $\varepsilon_{4}$ & $\varepsilon_{5}$ & $\varepsilon_{6}$ & $\varepsilon_{7}$ & $\varepsilon_{8}$ \\
\hline 3 & 2 & 0.910 & 0.584 & 0.429 & 0.339 & 0.280 & 0.238 & 0.207 \\
4 & 1 & 0.5 & 0.333 & 0.25 & 0.2 & 0.166 & 0.142 & 0.125 \\
5 & 0.666 & 0.347 & 0.235 & 0.178 & 0.143 & 0.119 & 0.103 & 0.090 \\
6 & 0.5 & 0.266 & 0.182 & 0.138 & 0.111 & 0.093 & 0.080 & 0.070
\end{tabular}

Moreover, the explicit expression of the first two constants is

$$
\varepsilon_{1}=\frac{2}{n-2}, \quad \varepsilon_{2}=\frac{2}{n}\left[\left(\frac{n}{n-2}\right)^{\frac{n-2}{2}}-1\right]^{-1},
$$

and $\varepsilon_{3}$ solves the equation

$$
\left[\mathcal{X}^{q}\left(\varepsilon_{3}\right)+\mathcal{W}\left(\varepsilon_{3}\right)\right]^{\frac{2}{q}}=\mathcal{X}^{2}\left(\varepsilon_{3}\right)+\frac{2}{q} \mathcal{W}\left(\varepsilon_{3}\right)
$$

where

$$
\mathcal{X}\left(\varepsilon_{3}\right)=\left(\frac{q}{2\left(\varepsilon_{3}+1\right)}\right)^{\frac{1}{q-2}}, \quad \mathcal{W}\left(\varepsilon_{3}\right)=1+\frac{1}{\varepsilon_{3}}\left(1-\frac{q}{2}\right), \quad q=\frac{2 n}{n-2} .
$$

Finally, if the dimension is $n=4$, we have $\varepsilon_{\ell}=\frac{1}{\ell}$ for every positive integer $\ell$.

We wish to remark that conditions $\left(\mathbf{K}_{\mathbf{1}}\right)$ and $\left(\mathbf{K}_{\mathbf{2}}\right)$ are crucial to obtain multiplicity results, but they can be weakened or overlooked when dealing with the existence of at least a G.S. with fast decay. Condition $\left(\mathbf{K}_{\mathbf{0}}\right)$ is a technical requirement which greatly simplifies the proof. The possibility of removing such a condition will be the object of forthcoming investigations.

As a first step in our analysis we also obtain the following existence result which does not require any integral or asymptotic condition.

Theorem 1.2 Assume that $K$ satisfies $\left(\mathbf{K}_{\mathbf{0}}\right)$ and (1.5) with $0<\varepsilon \leq \varepsilon_{1}:=\frac{2}{n-2}$, then Eq. (1.3) admits at least a G.S. with fast decay.

The symmetric condition $\left(\mathbf{K}_{\mathbf{0}}\right)$ allows to overcome the Pohozaev obstruction; furthermore the smallness condition is again quantitative and not of perturbative nature.

We emphasize that with a standard rescaling argument we can address Eq. (1.1) with more general bounded functions $K$. Indeed, let $v(r)$ be a solution of (1.3) where $K$ satisfies $0<$ 
$\underline{K} \leq K(r) \leq \bar{K}<\infty$, then $u(r)=\underline{K}^{(n-2) / 4} v(r)$ solves $\left(u^{\prime} r^{n-1}\right)^{\prime}+\mathscr{K}(r) r^{n-1} u^{\frac{n+2}{n-2}}=0$, where $\mathscr{K}(r):=\underline{K}^{-1} K(r)$ can be written in the form (1.5) with $\varepsilon=\bar{K} / \underline{K}-1$. So, we have the following.

Remark 1.3 Let $0<\underline{K} \leq K(r) \leq \bar{K}<\infty$ for any $r \geq 0$, then Theorems A and 1.2 keep on holding, simply by replacing the condition $0<\varepsilon<\varepsilon_{\ell}$ with $\bar{K} / \underline{K}<1+\varepsilon_{\ell}$.

Theorems 1.1, 1.2 and Remark 1.3 can be trivially generalized to embrace the slightly more general case of

$$
\Delta u+r^{\sigma}[1+\varepsilon k(|x|)] u^{q(\sigma)-1}=0, \text { where } q(\sigma)=2 \frac{n+\sigma}{n-2},
$$

and $\sigma>-2$. Notice that in this case we cannot apply directly [5, Theorem 2], so G.S. need not be radial.

Anyway, restricting to consider just radial G.S, we can reduce Eq. (1.9) to

$$
\left(u^{\prime} r^{n-1}\right)^{\prime}+r^{n-1+\sigma}[1+\varepsilon k(|x|)] u^{q(\sigma)-1}=0 .
$$

Then, we can prove a slightly more general version of Theorem A which has not appeared in literature previously, as far as we are aware.

Corollary 1.4 Theorem A continues to hold for Eq. (1.10) when $0<k(|x|)<1$. Furthermore, we can reprove Theorems 1.2 and 1.1 as well. We stress that in the equations defining $\varepsilon_{j}$ we must replace $q=q(0)$ by $q(\sigma)$. Consequently, all the values in the table 1.7 have to be slightly modified. In particular, we find

$$
\begin{aligned}
& \varepsilon_{1}=\frac{q-2}{2}=\frac{2+\sigma}{n-2}, \\
& \varepsilon_{2}=\frac{q-2}{q}\left[\left(\frac{q}{2}\right)^{\frac{2}{q-2}}-1\right]^{-1}=\frac{2+\sigma}{n+\sigma}\left[\left(\frac{n+\sigma}{n-2}\right)^{\frac{n-2}{2+\sigma}}-1\right]^{-1} .
\end{aligned}
$$

To conclude this incomplete review of the vast literature on the problem, we wish to draw to the reader's attention the interesting paper [35], where Wei and Yan prove that if $K(|x|)$ has a positive maximum there are infinitely many non-radial G.S. This result, together with $[9,15]$ and the present article, seems to suggest that the bubble tower phenomenon occurs in the presence of a critical point of $K(|x|)$, and it is made up by radial solutions if the critical point is a minimum and by non-radial ones if it is a maximum.

Ours proofs are developed through basic tools of phase plane analysis after passing from (1.3) to the two-dimensional dynamical system (2.2), via Fowler transformation. The problem of existence of G.S. with fast decay is then translated into a problem of existence of homoclinic trajectories. Following the outline of [9], we perform a shooting argument, within system (2.2), from the origin towards the isocline $\dot{x}=0$. The results are then obtained by combining some barrier sets constructed in Section 3 and an asymptotic result borrowed from $[9,10]$.

The paper is organized as follows. In Sect. 2, we introduce the Fowler transformation and we give some preliminary results. In Sect. 3, we sketch the geometrical construction on which the proof of our main results developed in Sect. 4 is based. In Sect. 5, we compute explicitly the values of the constants $\varepsilon_{\ell}$. In Appendix, we reprove [10, Theorem 1.6] for completeness. 


\section{Fowler transformation and invariant manifolds}

Let us introduce a classical change of variable, known as Fowler transformation, to convert Eq. (1.3) into a two-dimensional dynamical system. More precisely, by setting

$$
\begin{aligned}
x(t) & =u(r) r^{\alpha}, & & y(t)=\alpha u(r) r^{\alpha}+u^{\prime}(r) r^{\alpha+1}, \\
\alpha & =\frac{n-2}{2}, & & r=e^{t}, \quad \mathcal{K}(t)=K\left(e^{t}\right)
\end{aligned}
$$

we can rewrite (1.3) as the following system:

$$
\left(\begin{array}{l}
\dot{x} \\
\dot{y}
\end{array}\right)=\left(\begin{array}{cc}
0 & 1 \\
\alpha^{2} & 0
\end{array}\right)\left(\begin{array}{l}
x \\
y
\end{array}\right)+\left(\begin{array}{c}
0 \\
-\mathcal{K}(t) x^{q-1}
\end{array}\right),
$$

where "." denotes the differentiation with respect to $t$, and $q=\frac{2 n}{n-2}$.

Remark 2.1 If we start from (1.10), we obtain system (2.2) again, but the power $q$ is different, i.e. $q(\sigma)=2 \frac{n+\sigma}{n-2}$.

We collect here some notations that will be in force throughout the whole paper. Let $\boldsymbol{Q} \in \mathbb{R}^{2}$, we denote by $\boldsymbol{\phi}(t ; \tau, \boldsymbol{Q})$ the trajectory of (2.2) which is in $\boldsymbol{Q}$ at $t=\tau$. We denote by $u(r ; d)$ the regular solution of (1.3) such that $u(0 ; d)=d>0$ and $u^{\prime}(0 ; d)=0$, and by $\phi(t, d)=(x(t, d), y(t, d))$ the corresponding trajectory of (2.2). The origin is a critical point for (2.2) and the linearization of (2.2) at the origin has constant positive and negative eigenvalues, i.e. $\pm \alpha$, so the origin is a saddle. Moreover, from $[12, \S 13.4]$, we see that the non-autonomous system (2.2) admits unstable and stable leaves, i.e.

$$
\begin{aligned}
& W^{u}(\tau):=\left\{\boldsymbol{Q} \mid \lim _{t \rightarrow-\infty} \boldsymbol{\phi}(t ; \tau, \boldsymbol{Q})=(0,0)\right\}, \\
& W^{s}(\tau):=\left\{\boldsymbol{Q} \mid \lim _{t \rightarrow \infty} \boldsymbol{\phi}(t ; \tau, \boldsymbol{Q})=(0,0)\right\} .
\end{aligned}
$$

Namely, $W^{u}(\tau)$ and $W^{s}(\tau)$ are $C^{1}$ immersed one-dimensional manifolds.

Another way to construct $W^{u}(\tau)$ is to add the extra variable $z=e^{\varpi t}$, where $\varpi>0$, so that the system (2.2) can be rewritten as the equivalent autonomous three-dimensional system

$$
\left(\begin{array}{c}
\dot{x} \\
\dot{y} \\
\dot{z}
\end{array}\right)=\left(\begin{array}{ccc}
0 & 1 & 0 \\
\alpha^{2} & 0 & 0 \\
0 & 0 & \varpi
\end{array}\right)\left(\begin{array}{l}
x \\
y \\
z
\end{array}\right)+\left(\begin{array}{c}
0 \\
-\mathcal{K}\left(\frac{\ln (z)}{\varpi}\right) x^{q-1} \\
0
\end{array}\right) .
$$

It can be shown that if (2.4) is smooth, it admits a two-dimensional unstable manifold $\boldsymbol{W}^{\boldsymbol{u}}$ and that

$$
W^{u}(\tau)=\left\{\boldsymbol{Q} \in \mathbb{R}^{2} \mid(\boldsymbol{Q}, z(\tau)) \in \boldsymbol{W}^{\boldsymbol{u}}\right\} .
$$

This allows us to define $W^{u}(-\infty)=\left\{\boldsymbol{Q} \in \mathbb{R}^{2} \mid(\boldsymbol{Q}, 0) \in \boldsymbol{W}^{\boldsymbol{u}}\right\}$, which is the unstable manifold of the frozen autonomous system where $\mathcal{K} \equiv \mathcal{K}(0)$.

Since the flow of (2.2) is ruled by its linear part close to the origin, according to [22,25] and $[12, \S 13.4]$, we easily deduce the following properties of the unstable and stable leaves, respectively.

Remark 2.2 Assume that $\mathcal{K} \in C^{1}$ and it is bounded. Then,

$$
u(r ; d) \text { is a regular solution } \Longleftrightarrow \phi\left(\tau_{0}, d\right)=Q \in W^{u}\left(\tau_{0}\right) .
$$

Moreover, $W^{u}(\tau)$ is tangent in the origin to the line $y=\alpha x$, for any $\tau \in \mathbb{R}$, and it depends smoothly on $\tau$, i.e., let $L$ be a segment which intersects $W^{u}\left(\tau_{0}\right)$ transversally in a point 
$\boldsymbol{Q}\left(\tau_{0}\right)$, then there is a neighbourhood $I$ of $\tau_{0}$ such that $W^{u}(\tau)$ intersects $L$ in a point $\boldsymbol{Q}(\tau)$ for any $\tau \in I$, and $Q(\tau) \in C^{1}$.

Furthermore, if (2.4) is smooth for $z=0$ too (e.g. if $\left(\mathbf{K}_{\mathbf{2}}\right)$ holds and $0<\varpi<l$ ), the smoothness property of $W^{u}(\tau)$ is extended to $\tau_{0}=-\infty$, i.e. to the system (2.4) restricted to $z=0$, see $[23, \S 2.2]$ for more details.

Similarly, fast decay solutions correspond to trajectories of $W^{s}(\tau)$.

Remark 2.3 Assume that $\mathcal{K} \in C^{1}$ and it is bounded. Let $\phi\left(t ; \tau_{0}, \boldsymbol{Q}\right)$ be the trajectory of (2.2) corresponding to the solution $u$ of (1.3). Then,

$$
u(r) \text { is a fast decay solution } \Longleftrightarrow \boldsymbol{\phi}\left(\tau_{0} ; \tau_{0}, \boldsymbol{Q}\right)=\boldsymbol{Q} \in W^{s}\left(\tau_{0}\right) .
$$

Moreover, $W^{s}(\tau)$ is tangent in the origin to the line $y=-\alpha x$, for any $\tau \in \mathbb{R}$, and it depends smoothly on $\tau$.

We stress that the manifold $W^{u}(\tau)$ (as well as $W^{s}(\tau)$ ) is divided by the origin in two connected components: one which leaves the origin and enters $x>0$, and the other that enters $x<0$. Since we are just interested in positive solutions, abusing the notation, we let $W^{u}(\tau)$ and $W^{s}(\tau)$ stand for the branches of the leaves which depart from the origin and enter in $x>0$.

Remark 2.4 Assume that $\mathcal{K} \in C^{1}$ and it is bounded. Fix $\tau \in \mathbb{R}$, and let $\boldsymbol{Q}(d) \in W^{u}(\tau)$ be such that $\phi(\tau, d)=\boldsymbol{Q}(d)$, for every $d \geq 0$. Then, the function $\boldsymbol{Q}:[0,+\infty) \rightarrow W^{u}(\tau)$ is a smooth (bijective) parametrization of $W^{u}(\tau)$ and $\boldsymbol{Q}(0)=(0,0)$.

We refer to [13, Lemma 2.10] for the proof of Remark 2.4. Let us also notice that, in a similar way, the stable leave $W^{s}(\tau)$ can be parametrized directly by $c:=\lim _{r \rightarrow \infty} u(r) r^{n-2}$.

Remark 2.5 Any regular solution $u(r ; d)$ is decreasing until its first zero; so Ground States are monotone decreasing.

For an easy proof of the remark, we refer to [32, Lemma 3.7].

Assumption $\left(\mathbf{K}_{\mathbf{0}}\right)$ guarantees that $\mathcal{K}$ is even, i.e. $\mathcal{K}(-t)=\mathcal{K}(t)$ for any $t \in \mathbb{R}$. Hence, if $\boldsymbol{\phi}(t, d)=(x(t), y(t))$ solves $(2.2)$ and $y(0)=0$, then $x(t)$ is even and $y(t)$ is odd. Thus, we get the following.

Remark 2.6 Assume $\left(\mathbf{K}_{\mathbf{0}}\right)$. If $\boldsymbol{\phi}(t, d)$ is such that $x(t, d)>0$ for $t \leq 0$, and $y(0, d)=0$, then $u(r ; d)$ is a monotone decreasing G.S. with fast decay.

To illustrate the main ideas of the proofs of Theorem 1.2 and Theorem A, we enumerate some results which will be proved in Sect. 4.

Taking into account that the origin is a critical point and that $W^{u}(\tau)$ is tangent in the origin to the line $y=\alpha x$, we easily deduce that $x(t, d)$ is strictly increasing and, consequently, $y(t, d)$ is strictly positive for $t$ in a neighbourhood of $-\infty$, for any $d>0$.

For any $\ell \in \mathbb{N}, \ell \geq 1$ we define the sets

$$
I_{\ell}:=\{d>0 \mid y(t, d) \text { has at least } \ell \text { zeroes for } t \in \mathbb{R}\} .
$$

We denote by $T_{1}(d)$ and $T_{\ell}(d)$, respectively, the first and the $\ell$ th zero of $y(t, d)=\dot{x}(t, d)$, i.e.

$$
\begin{array}{ll}
T_{1}(d):=\min \{t \in \mathbb{R} \mid y(t, d)=0\}, & R_{1}(d):=e^{T_{1}(d)}, \\
T_{\ell}(d):=\min \left\{t>T_{\ell-1}(d) \mid y(t, d)=0\right\}, & R_{\ell}(d):=e^{T_{\ell}(d)} .
\end{array}
$$


Remark 2.7 Assume (1.5) and $0<\varepsilon \leq \varepsilon_{1}$. Then, there is $D_{1}>0$ such that $R_{1}(d)>1$ for every $d \in\left(0, D_{1}\right)$.

This Remark is proved in Section 4 as an easy consequence of Remark 4.3.

In Sect. 4 (Lemma 4.4) and in Appendix, we reprove for completeness the following result, which is a consequence of $[9,10]$.

Proposition 2.8 [10, Theorem 1.6], [9, Lemma 2.2] Assume $\left(\mathbf{K}_{\mathbf{1}}\right)$ and $\left(\mathbf{K}_{\mathbf{2}}\right)$. Then, for any fixed $\ell \in \mathbb{N}, \ell \geq 1$, and for any $\rho>0$ there is $d_{\ell} \in I_{\ell}$ such that $R_{\ell}\left(d_{\ell}\right)<\rho$.

Under very mild conditions, we can show the continuity of $R_{1}(d)$.

Proposition 2.9 Assume (1.5) with $0<\varepsilon \leq \varepsilon_{1}$, then $I_{1}=(0,+\infty)$ and $R_{1}(d)$ is continuous in $I_{1}$. Furthermore, $\lim _{d \rightarrow+\infty} R_{1}(d)=0$ and $\lim _{d \rightarrow 0} R_{1}(d)=+\infty$.

Proposition 2.9 is restated in a dynamical context and proved in Sect. 4 as Lemma 4.9. From Proposition 2.9, we find $d_{1}^{*}>0$ such that $R_{1}\left(d_{1}^{*}\right)=1$; hence, if $\left(\mathbf{K}_{\mathbf{0}}\right)$ holds, $\boldsymbol{\phi}\left(t, d_{1}^{*}\right)$ is a homoclinic trajectory for (2.2) such that $x(t, d)$ is positive and increasing for $t \leq 0$ and positive and decreasing for $t \geq 0$. Then, Theorem 1.2 immediately follows from Remark 2.6.

To prove the continuity of $R_{j}(d)$ for $j \geq 2$, we need to require $\left(\mathbf{K}_{\mathbf{1}}\right)$ and to develop an articulated barrier argument which guarantees that the flow $\phi(t, d)$ intersects the $x$-axis transversally.

Proposition 2.10 Assume $\left(\mathbf{K}_{\mathbf{1}}\right)$ and (1.5) with $0<\varepsilon \leq \varepsilon_{\ell}$, where $\varepsilon_{\ell}$ is the computable constant given by Lemma 3.7 below. If $R_{j}(d) \leq 1$, then $R_{j}$ is continuous for any $j=1, \ldots, \ell$.

Let us sketch the proof of Theorem A, see Sect. 4 for a full fledged argument. Fix $\ell \in$ $\mathbb{N}$, assume $\left(\mathbf{K}_{\mathbf{0}}\right)-\left(\mathbf{K}_{\mathbf{1}}\right)-\left(\mathbf{K}_{\mathbf{2}}\right)$ and (1.5) with $0<\varepsilon<\varepsilon_{\ell}$. According to Proposition 2.8, Remark 2.7 and Proposition 2.10, for any $j=1, \ldots, \ell$ there is at least a value $d_{j}^{*} \in I_{j}$ such that $R_{j}\left(d_{j}^{*}\right)=1$. Hence, from Remark 2.6 we immediately infer that $\phi\left(t, d_{j}^{*}\right)$ is a homoclinic trajectory for (2.2) such that $x\left(t, d_{j}^{*}\right)$ is positive for any $t \in \mathbb{R}$ and $y\left(t, d_{j}^{*}\right)$ has exactly $(2 j-1)$ zeroes. This would complete the proof of Theorem A.

We emphasize that, in this paper as well as in [9], the simplicity of the zeros of $y(t, d)$ is crucial to obtain the continuity of the function $R_{j}$, i.e. Proposition 2.10. The contribution here with respect to [9] lies in the fact that the perturbative result in [9] gives no information on the actual size of the values $\varepsilon_{j}$ and no clue on how to compute them.

\section{Some geometrical constructions: meaning of $\varepsilon_{1}$ and definition of $\varepsilon_{\ell}$}

Define

$$
\mathcal{H}(x, y, t):=\frac{y^{2}}{2}-\alpha^{2} \frac{x^{2}}{2}+\mathcal{K}(t) \frac{x^{q}}{q}, \quad \text { with } q:=\frac{2 n}{n-2} .
$$

If we evaluate $\mathcal{H}$ along a solution $(x(t), y(t))$ of (2.2), we obtain the associated Pohozaev type energy $\mathcal{H}(x(t), y(t), t)$, whose derivative with respect to $t$ satisfies

$$
\frac{\mathrm{d}}{\mathrm{d} t} \mathcal{H}(x(t), y(t), t)=\frac{x(t)^{q}}{q} \frac{\mathrm{d} \mathcal{K}(t)}{\mathrm{d} t} .
$$

We immediately observe that if $\left(\mathbf{K}_{\mathbf{1}}\right)$ holds, then the function $\mathcal{H}$ is decreasing along the trajectories defined on negative values of $t$.

Moreover, if $u(r ; d)$ is a regular solution, then the corresponding trajectory $\phi(t, d)$ satisfies $\lim _{t \rightarrow-\infty} \mathcal{H}(\phi(t, d), t)=0$, since $\mathcal{H}(0,0, t) \equiv 0$. So, we easily get the following lemma. 
Lemma 3.1 Assume $\left(\mathbf{K}_{\mathbf{1}}\right)$. Then, $\mathcal{H}(x(t, d), y(t, d), t)<0$ for any $t \leq 0$ and $d>0$.

Let us now consider the frozen autonomous system (2.2) defined by setting $\mathcal{K}(t) \equiv 1+c$ for any $t \in \mathbb{R}$, for a certain constant $c \geq 0$, i.e.

$$
\left(\begin{array}{c}
\dot{x} \\
\dot{y}
\end{array}\right)=\left(\begin{array}{cc}
0 & 1 \\
\alpha^{2} & 0
\end{array}\right)\left(\begin{array}{l}
x \\
y
\end{array}\right)+\left(\begin{array}{c}
0 \\
-(c+1) x^{q-1}
\end{array}\right) .
$$

We introduce the corresponding energy function

$$
H_{c}(x, y):=\frac{y^{2}}{2}+G_{c}(x), \quad \text { where } \quad G_{c}(x)=-\alpha^{2} \frac{x^{2}}{2}+(1+c) \frac{x^{q}}{q} .
$$

Notice that if $(x(t), y(t))$ is a solution of (2.2), then

$$
\frac{\mathrm{d}}{\mathrm{d} t} H_{c}(x(t), y(t))=y(t) x(t)^{q-1}(1+c-\mathcal{K}(t)) .
$$

System (3.3) admits a homoclinic orbit

$$
\Gamma_{c}=\left\{(x, y) \mid H_{c}(x, y)=0, x>0\right\} .
$$

Lemma 3.2 Assume (1.5) and $\left(\mathbf{K}_{\mathbf{1}}\right)$. Then, the trajectory $\boldsymbol{\phi}(t, d)$ of system (2.2) belongs to the region enclosed by $\Gamma_{0}$ for every $t \leq 0$ and $d>0$. In particular, $x(t, d)>0$ for any $t \leq 0$.

Proof Assumption (1.5) ensures that $\mathcal{K}(t) \geq 1$ for every $t \leq 0$. Thus, combining definitions (3.1) and (3.4) with Lemma 3.1, we immediately deduce that

$$
H_{0}(x(t, d), y(t, d)) \leq \mathcal{H}(x(t, d), y(t, d), t)<0, \quad \forall t \leq 0 .
$$

Remark 3.3 The homoclinic orbit $\Gamma_{c_{1}}$ belongs to the region enclosed by $\Gamma_{c_{2}}$, whenever $c_{1}>$ $c_{2}$.

System (3.3) admits a unique critical point $\boldsymbol{P}^{*}(c)=\left(P_{x}^{*}(c), 0\right)$ such that $P_{x}^{*}(c)>0$, i.e.

$$
P_{x}^{*}(c)=\left(\frac{\alpha^{2}}{c+1}\right)^{\frac{1}{q-2}}=\left(\frac{(n-2)^{2}}{4(c+1)}\right)^{\frac{n-2}{4}} .
$$

Remark 3.4 Set $G_{c}^{\min }:=G_{c}\left(P_{x}^{*}(c)\right)$, the minimum of $G_{c}$ in the interval $[0,+\infty)$. Fix $g \in$ $\left(G_{c}^{\min }, 0\right)$; then the equation $G_{c}(x)=g$ admits exactly two positive solutions $x_{1, c}(g)$ and $x_{2, c}(g)$ such that $0<x_{1, c}(g)<P_{x}^{*}(c)<x_{2, c}(g)$. We denote by $A_{1}(c)$ the unique positive solution of the equation $G_{c}(x)=0$, i.e.

$$
A_{1}(c):=x_{2, c}(0)=\left(\frac{\alpha^{2} q}{2(c+1)}\right)^{\frac{1}{q-2}}=\left(\frac{q}{2}\right)^{\frac{1}{q-2}} P_{x}^{*}(c)=\left(\frac{(n-2) n}{4(c+1)}\right)^{\frac{n-2}{4}} .
$$

Remark 3.5 The functions $x_{1, c}(g)$ and $x_{2, c}(g)$ are, respectively, decreasing and increasing functions with respect to the energy $g \in\left(G_{c}^{\mathrm{min}}, 0\right]$. In particular, $\boldsymbol{A}_{\mathbf{1}}(c)=\left(A_{1}(c), 0\right)$ is the right extremal of $\Gamma_{c}$.

We set $\varepsilon_{1}=\frac{q-2}{2}=\frac{2}{n-2}=\frac{1}{\alpha}$, so that, from (3.7) and (3.8), we find

$$
\begin{gathered}
A_{1}\left(\varepsilon_{1}\right)=P_{x}^{*}(0), \\
P_{x}^{*}(0) \leq A_{1}(\varepsilon) \Longleftrightarrow 0<\varepsilon \leq \varepsilon_{1} .
\end{gathered}
$$




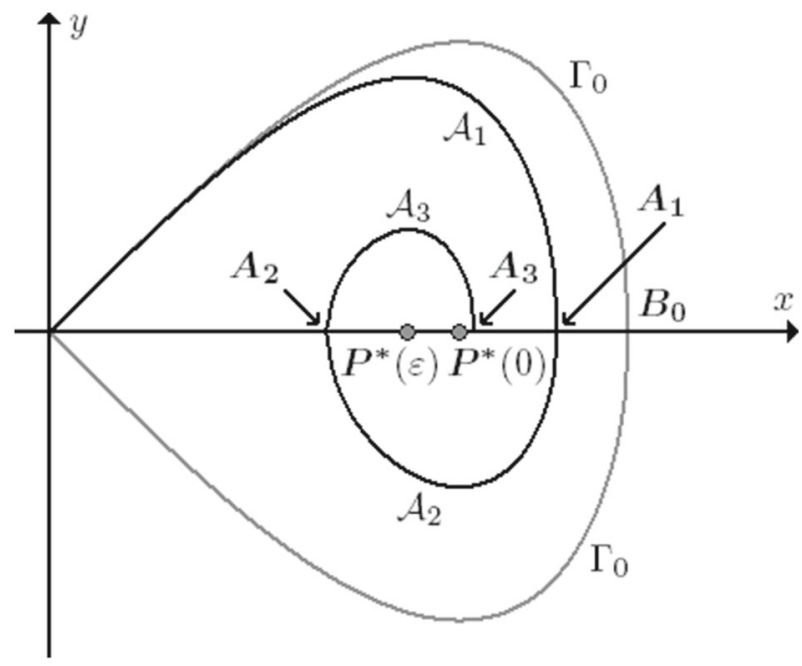

Fig. 1 The construction of the path $\gamma$ consisting of the sets $\mathcal{A}_{j}$

We are now going to define a spiral-like path $\gamma$ inside the region bounded by $\Gamma_{0}$, cf. Fig. 1. The path $\boldsymbol{\gamma}$ will rotate several times around the points $\boldsymbol{P}^{*}(\varepsilon)$ and $\boldsymbol{P}^{*}(0)$ provided that $\varepsilon$ is sufficiently small, as described in Lemma 3.7 below. Such a path will allow to locate the solutions of the non-autonomous system (2.2) satisfying $(x(t), y(t)) \rightarrow(0,0)$ as $t \rightarrow-\infty$, cf. Fig. 2 and Lemma 4.6 below.

We emphasize that the spiral-like path $\gamma$ depends on $\varepsilon$, but we leave this dependence unsaid for simplicity. The path $\boldsymbol{\gamma}$ is made up by the level curves of $H_{\varepsilon}$ in the half-plane $\{y \geq 0\}$ and by the level curves of $H_{0}$ in the half-plane $\{y \leq 0\}$. The continuity of the path is guaranteed by the proper choice of the levels.

Let us introduce the curve

$$
\mathcal{A}_{1}:=\left\{(x, y) \mid H_{\varepsilon}(x, y)=0, x>0, y \geq 0\right\}=\Gamma_{\varepsilon} \cap\{(x, y) \mid y \geq 0\},
$$

joining the origin to the point $\boldsymbol{A}_{\mathbf{1}}(\varepsilon)=\left(A_{1}(\varepsilon), 0\right)$. We set

$$
\mathcal{H}_{1}:=H_{0}\left(\boldsymbol{A}_{\mathbf{1}}\right)=H_{\varepsilon}\left(\boldsymbol{A}_{\mathbf{1}}\right)-\frac{\varepsilon}{q} A_{1}^{q}=-\frac{\varepsilon}{q} A_{1}^{q} .
$$

Then, for any $0<\varepsilon \leq \varepsilon_{1}$ we define

$$
\mathcal{A}_{2}=\left\{(x, y) \mid H_{0}(x, y)=\mathcal{H}_{1}, \quad x>0, y \leq 0\right\},
$$

and $\boldsymbol{A}_{\mathbf{2}}=\left(A_{2}, 0\right)$ as the left extremal of $\mathcal{A}_{2}$, i.e. $A_{2}=x_{1,0}\left(\mathcal{H}_{1}\right)$.

Remark 3.6 For any $0 \leq \varepsilon \leq \varepsilon_{1}$, the functions $A_{1}=A_{1}(\varepsilon)$ and $\mathcal{H}_{1}=\mathcal{H}_{1}(\varepsilon)$ are both continuous and decreasing, while $A_{2}=A_{2}(\varepsilon)$ is continuous and increasing. Furthermore, $\mathcal{A}_{2}$ belongs to the region enclosed by $\Gamma_{\varepsilon}$.

Proof The properties of $A_{1}(\varepsilon)$ and $\mathcal{H}_{1}(\varepsilon)$ follow from (3.8) and (3.11). Since $\mathcal{H}_{1}(\varepsilon)$ is decreasing, from Remark 3.5 we find that $A_{2}(\varepsilon)=x_{1,0}\left(\mathcal{H}_{1}(\varepsilon)\right)$ is increasing.

Since $P_{x}^{*}(\varepsilon)$ is descreasing, we deduce that $P_{x}^{*}-A_{2}$ is a continuous decreasing function satisfying

$$
P_{x}^{*}(0)-A_{2}(0)=P_{x}^{*}(0)>0, \quad P_{x}^{*}\left(\varepsilon_{1}\right)-A_{2}\left(\varepsilon_{1}\right)=P_{x}^{*}\left(\varepsilon_{1}\right)-P_{x}^{*}(0)<0,
$$


which guarantees the existence of $\varepsilon_{2} \in\left(0, \varepsilon_{1}\right)$ such that

$$
A_{2}\left(\varepsilon_{2}\right)=P_{x}^{*}\left(\varepsilon_{2}\right) \text {. }
$$

(We will show in Sect. 5 how to compute $\varepsilon_{2}$ ). Now, assume $0<\varepsilon<\varepsilon_{2}$, so that $A_{2}<P_{x}^{*}(\varepsilon)$ : taking into account (3.11), we set

$$
\mathcal{H}_{2}:=H_{\varepsilon}\left(\boldsymbol{A}_{\mathbf{2}}\right)=H_{0}\left(\boldsymbol{A}_{\mathbf{2}}\right)+\frac{\varepsilon}{q} A_{2}^{q}=-\frac{\varepsilon}{q}\left(A_{1}^{q}-A_{2}^{q}\right)<0 .
$$

We define

$$
\mathcal{A}_{3}=\left\{(x, y) \mid H_{\varepsilon}(x, y)=\mathcal{H}_{2}, \quad x>0, y \geq 0\right\},
$$

and $\boldsymbol{A}_{\mathbf{3}}=\left(A_{3}, 0\right)$ as the right extremal of $\mathcal{A}_{3}$. In particular, $A_{3}=x_{2, \varepsilon}\left(\mathcal{H}_{2}\right)$. Combining Remarks 3.5 and 3.6, we deduce that $\mathcal{H}_{2}(\varepsilon)$ and, consequently, $A_{3}(\varepsilon)$ are both continuous and decreasing for $\varepsilon \in\left(0, \varepsilon_{2}\right]$. Moreover, from (3.13) and (3.9), we deduce that $A_{3}\left(\varepsilon_{2}\right)=$ $P_{x}^{*}\left(\varepsilon_{2}\right)<P_{x}^{*}(0)$ and $\lim _{\varepsilon \rightarrow 0} A_{3}(\varepsilon)=x_{2,0}(0)=A_{1}(0)>P_{x}^{*}(0)$, which ensures the existence of $\varepsilon_{3} \in\left(0, \varepsilon_{2}\right)$ such that

$$
A_{3}\left(\varepsilon_{3}\right)=P_{x}^{*}(0) \text {. }
$$

For $\varepsilon<\varepsilon_{3}$ we have $A_{3}>P_{x}^{*}(0)$, and we can construct the set

$$
\mathcal{A}_{4}=\left\{(x, y) \mid H_{0}(x, y)=\mathcal{H}_{3}, \quad x>0, y \leq 0\right\},
$$

where $\mathcal{H}_{3}:=H_{0}\left(\boldsymbol{A}_{\mathbf{3}}\right)=H_{\varepsilon}\left(\boldsymbol{A}_{\mathbf{3}}\right)-\frac{\varepsilon}{q} A_{3}^{q}=\mathcal{H}_{2}-\frac{\varepsilon}{q} A_{3}^{q}=-\frac{\varepsilon}{q}\left(A_{1}^{q}-A_{2}^{q}+A_{3}^{q}\right)<0$. Finally, we locate the point $\boldsymbol{A}_{\mathbf{4}}=\left(A_{4}, 0\right)$ as the left extremal of $\mathcal{A}_{4}$, so that $A_{4}=x_{1,0}\left(\mathcal{H}_{3}\right)$. Iterating such a procedure, we have the following lemma which summarizes the situation, see Fig. 1.

Lemma 3.7 There exists a decreasing sequence of positive values $\left(\varepsilon_{\ell}\right)_{\ell \in \mathbb{N}}$ with the following property: for every $\varepsilon<\varepsilon_{\ell}$, we can construct the sets $\mathcal{A}_{1}, \ldots, \mathcal{A}_{\ell}$ as follows:

$$
\begin{aligned}
& \mathcal{A}_{2 i+1}=\left\{(x, y) \mid H_{\varepsilon}(x, y)=\mathcal{H}_{2 i}, x>0, y \geq 0\right\}, \quad \\
& \mathcal{A}_{2 i+2}=\left\{(x, y) \mid H_{0}(x, y)=\mathcal{H}_{2 i+1}, x>0, y \leq 0\right\}, \quad(i \in \mathbb{N})
\end{aligned}
$$

where $\mathcal{H}_{0}=0$,

$$
\mathcal{H}_{j}=\frac{\varepsilon}{q} \sum_{i=1}^{j}(-1)^{i} A_{i}^{q},
$$

with $A_{2 i+1}=x_{2, \varepsilon}\left(\mathcal{H}_{2 i}\right), A_{2 i+2}=x_{1,0}\left(\mathcal{H}_{2 i+1}\right)$. Moreover,

$$
\begin{aligned}
& A_{2}<\cdots<A_{2 i}<A_{2 i+2}<\cdots<P_{x}^{*}(\varepsilon)<P_{x}^{*}(0)< \\
& \quad<\cdots<A_{2 i+3}<A_{2 i+1}<\cdots<A_{1} .
\end{aligned}
$$

We can glue together the sets $\mathcal{A}_{1}, \ldots, \mathcal{A}_{\ell}$ and draw a spiral-like path $\boldsymbol{\gamma}$ which rotates around the points $\boldsymbol{P}^{*}(\varepsilon)$ and $\boldsymbol{P}^{*}(0)$.

Finally, if we choose a critical value $\varepsilon=\varepsilon_{\ell}$, we can construct the sets $\mathcal{A}_{1}, \ldots, \mathcal{A}_{\ell}$ using the definitions above, with the only difference that $A_{\ell}=P_{x}^{*}(0)$ if $\ell$ is odd and $A_{\ell}=P_{x}^{*}\left(\varepsilon_{\ell}\right)$ if $\ell$ is even.

The function $A_{j}=A_{j}(\varepsilon)$ is continuous and decreasing if $j$ is odd, while it is increasing when $j$ is even; $\mathcal{H}_{j}=\mathcal{H}_{j}(\varepsilon)$ is continuous and decreasing, and $\mathcal{H}_{j+2}<\mathcal{H}_{j}$.

Remark 3.8 Note that $\mathcal{H}_{2 j+1}=H_{0}\left(\boldsymbol{A}_{\mathbf{2} j+1}\right)$ and $\mathcal{H}_{2 j}=H_{\varepsilon}\left(\boldsymbol{A}_{\mathbf{2} j+\mathbf{1}}\right)$, which implies that $A_{2 i+1}=x_{2,0}\left(\mathcal{H}_{2 i+1}\right)$ and $A_{2 i+2}=x_{1, \varepsilon}\left(\mathcal{H}_{2 i+2}\right)$. Finally, observe that $\boldsymbol{\gamma} \subseteq \Gamma_{\varepsilon} \subset \Gamma_{0}$.

The computation of the values $\varepsilon_{\ell}$ is postponed to Sect. 5 . 


\section{Proofs}

In the previous section, we have constructed the path $\gamma$ gluing together trajectories of the autonomous systems (3.3). Now, we turn to consider the non-autonomous problem (2.2). The first step is to locate the initial branch of the unstable manifold $W^{u}(\tau)$ : this will be enough to prove Theorem 1.2. Then, to obtain the multiplicity result, Theorem A, we will use the set $\gamma$ to control the behaviour of the solutions of (2.2) for $t \leq 0$.

Having in mind (3.4) and (3.8), we define

$$
\begin{aligned}
& \mathcal{B}_{1}=\left\{(x, y) \mid H_{0}(x, y)=0, x>0, y>0\right\} \subset \Gamma_{0}, \\
& L_{1}=\left\{(x, 0) \mid A_{1}(\varepsilon) \leq x \leq A_{1}(0)\right\}, \\
& E_{1}=\left\{(x, y) \mid H_{0}(x, y) \leq 0 \leq H_{\varepsilon}(x, y), x \geq 0, y \geq 0\right\} .
\end{aligned}
$$

Notice that $E_{1}$ is the set enclosed by $\Gamma_{0}$ and $\Gamma_{\varepsilon}$ in the half-plane $\{y \geq 0\}$.

We aim to prove the following.

Lemma 4.1 Assume (1.5) with $0<\varepsilon \leq \varepsilon_{1}$. Then, for any $\tau \in \mathbb{R}$ there is $\boldsymbol{\xi}_{\mathbf{1}}(\tau)=\left(\xi_{1}(\tau), 0\right)$ such that $\xi_{1}(\tau) \in\left[W^{u}(\tau) \cap L_{1}\right]$ and the connected branch $\tilde{W}^{u}(\tau)$ of the manifold $W^{u}(\tau)$ between the origin and $\xi_{1}(\tau)$ lies in $E_{1}$.

In order to prove Lemma 4.1, we need the following result.

Lemma 4.2 Assume (1.5), then the flow of (2.2) on $\mathcal{A}_{1}$ and $\mathcal{B}_{1}$ points towards the interior of $E_{1}$. Assume also $0<\varepsilon \leq \varepsilon_{1}$, then the flow of (2.2) on $L_{1}$ points towards the exterior of $E_{1}$ for any $t \in \mathbb{R}$. In particular, if $\boldsymbol{Q} \in L_{1}$, then $\dot{y}(t ; t, \boldsymbol{Q})<0$.

Proof From (3.5) and (1.5), we get

$$
\frac{\mathrm{d}}{\mathrm{d} t} H_{0}(x(t), y(t))<0<\frac{\mathrm{d}}{\mathrm{d} t} H_{\varepsilon}(x(t), y(t)),
$$

for every solution $(x(t), y(t))$ of (2.2) with $x(t)>0, y(t)>0$. This proves the first assertion of the lemma.

Assume now $0<\varepsilon \leq \varepsilon_{1}$ and consider a solution ( $\left.x(t), y(t)\right)$ of (2.2) with $\left(x\left(t_{0}\right), y\left(t_{0}\right)\right) \in L_{1}$. Thus, from (1.5) and (3.8) we get

$$
\mathcal{K}\left(t_{0}\right) x\left(t_{0}\right)^{q-2}>A_{1}(\varepsilon)^{q-2}=\frac{\alpha^{2} q}{2(\varepsilon+1)} \geq \frac{\alpha^{2} q}{2\left(\varepsilon_{1}+1\right)}=\alpha^{2},
$$

which implies

$$
\dot{y}\left(t_{0}\right)=x\left(t_{0}\right)\left(\alpha^{2}-\mathcal{K}\left(t_{0}\right) x\left(t_{0}\right)^{q-2}\right)<0 .
$$

This completes the proof of the lemma.

Proof of Lemma 4.1 We just sketch the proof inspired by Ważewski's principle; we refer to [16, Theorem 3.3], see also [19, Lemma 3.5], [23, Lemma 6.3] for more details.

Fix $\tau \in \mathbb{R}$. We claim that $L_{1} \cap W^{u}(\tau) \neq \emptyset$. Consider $\boldsymbol{Q} \in L_{1} \backslash W^{u}(\tau)$. Taking into account Lemma 4.2 and the absence of invariant sets in the interior of $E_{1}$, we define $\mathcal{T}(\boldsymbol{Q})$ as the value in $(-\infty, \tau]$ such that $\boldsymbol{\phi}(t ; \tau, \boldsymbol{Q}) \in E_{1}$ for every $t \in(\mathcal{T}(\boldsymbol{Q}), \tau)$ and $\boldsymbol{\phi}(\mathcal{T}(\boldsymbol{Q}) ; \tau, \boldsymbol{Q}) \in$ $\left(\mathcal{A}_{1} \cup \mathcal{B}_{1}\right)$. Combining (4.2) with the continuity of the flow, it is easy to check that the sets

$$
\begin{aligned}
& L_{\mathcal{A}}:=\left\{\boldsymbol{Q} \in L_{1} \mid \boldsymbol{\phi}(\mathcal{T}(\boldsymbol{Q}) ; \tau, \boldsymbol{Q}) \in \mathcal{A}_{1}\right\}, \\
& L_{\mathcal{B}}:=\left\{\boldsymbol{Q} \in L_{1} \mid \boldsymbol{\phi}(\mathcal{T}(\boldsymbol{Q}) ; \tau, \boldsymbol{Q}) \in \mathcal{B}_{1}\right\}
\end{aligned}
$$


are open. Furthermore, observe that $\left(A_{1}(\varepsilon), 0\right) \in L_{\mathcal{A}}$ and $\left(A_{1}(0), 0\right) \in L_{\mathcal{B}}$; from a connection argument, it follows that there is $\xi_{1}(\tau) \in L_{1}, \xi_{1}(\tau) \notin L_{\mathcal{A}} \cup L_{\mathcal{B}}$. Then, according to Lemma 4.2, we easily deduce that $\phi\left(t ; \tau, \xi_{1}(\tau)\right) \in E_{1}$ for any $t<\tau$, and $\lim _{t \rightarrow-\infty} \phi\left(t ; \tau, \boldsymbol{\xi}_{\mathbf{1}}(\tau)\right)=(0,0)$. Therefore, $\boldsymbol{\xi}_{\mathbf{1}}(\tau) \in W^{u}(\tau)$, which proves the claim.

Consider a continuous path $\sigma:[0,1] \rightarrow \mathbb{R}$ joining $\mathcal{A}_{1}$ and $\mathcal{B}_{1}$ with $\sigma(0) \in \mathcal{A}_{1}$ and $\sigma(1) \in \mathcal{B}_{1}$. We could adopt the argument above, with $L_{1}$ replaced by $\sigma$, to prove the existence of $s \in(0,1)$ such that $\sigma(s) \in W^{u}(\tau)$. Then, applying Lemma 4 in [34] we conclude that the set $W^{u}(\tau)$ defined as in (2.3) contains a compact connected set $\tilde{W}^{u}(\tau)$ which contains the origin, $\xi_{1}(\tau) \in L_{1}$ and such that $\tilde{W}^{u}(\tau) \subset E_{1}$. Furthermore, using the classical results in $[12, \S 13.4]$, we see that $\tilde{W}^{u}(\tau)$ and $W^{u}(\tau)$ are indeed one-dimensional immersed manifolds, see also [23, Lemma 6.5].

Combining the previous results with Remark 2.4, we can state the following.

Remark 4.3 Assume (1.5) with $0<\varepsilon \leq \varepsilon_{1}$, fix $\tau \in \mathbb{R}$ and let $d_{*}(\tau)>0$ be such that $\boldsymbol{\phi}\left(\tau, d_{*}(\tau)\right)=\boldsymbol{\xi}_{\mathbf{1}}(\tau)$, then $\boldsymbol{\phi}(\tau, d) \in \tilde{W}^{u}(\tau)$ for any $d \leq d_{*}(\tau)$. In fact, the map $\phi(\tau, \cdot)$ : $\left[0, d_{*}(\tau)\right] \rightarrow E_{1}$ is a smooth parametrization of $\tilde{W}^{u}(\tau)$. Furthermore, by construction, $\boldsymbol{\phi}(t, d) \in \tilde{W}^{u}(t) \subset E_{1}$ for any $t \leq \tau$ and any $0<d \leq d_{*}(\tau)$.

According to definition (2.6), we notice that $T_{1}\left(d_{*}(\tau)\right)=\tau$, whence $T_{1}(d)>0$ for any $d<D_{1}=d_{*}(0)$. Thus, Remark 2.7 immediately follows.

We are now ready to prove Theorem 1.2.

Proof of Theorem 1.2 Setting $\tau=0$ and applying Lemma 4.1 and Remark 4.3, we see that $\phi\left(t, d_{*}(0)\right) \in E_{1}$ for any $t<0$. In addition, $x\left(t, d_{*}(0)\right)>0$ and $y\left(t, d_{*}(0)\right)>0$ for $t<0$, and $y\left(0, d_{*}(0)\right)=0$. Thanks to assumption $\left(\mathbf{K}_{\mathbf{0}}\right)$, we can apply Remark 2.6 to deduce that $u\left(r ; d_{*}(0)\right)$ is a G.S. with fast decay and $x\left(t, d_{*}(0)\right)$ has a unique critical point which is a positive maximum.

Lemma 4.4 [10, Theorem 1.6], [9, Lemma 2.2] Assume conditions $\left(\mathbf{K}_{\mathbf{1}}\right)-\left(\mathbf{K}_{\mathbf{2}}\right)$ and fix $\rho \in$ $(0,1)$. For any positive integer $\ell$, there is $d_{\ell}$ such that $x\left(t, d_{\ell}\right)>0$ and $y\left(t, d_{\ell}\right)$ has at least $\ell$ non-degenerate zeroes for $t<\ln (\rho)$.

As a consequence, $T_{\ell}\left(d_{\ell}\right)<\ln (\rho)<0$.

Notice that the last assertion gives Proposition 2.8. The proof of Lemma 4.4 is far from being trivial, and it is reproved in Appendix for completeness, following the original idea of [10, Theorem 1.6]. In fact, from Proposition 2.8 we easily get the existence of a trajectory of (2.2) having the whole set $\Gamma_{\varepsilon}$ as $\alpha$-limit set. We emphasize that if $l \geq \frac{n-2}{2}$ in $\left(\mathbf{K}_{\mathbf{2}}\right)$ such a trajectory does not exist, see [10, Theorem 1.1].

We are now interested in showing the continuity of the maps $T_{\ell}(d)$ and $R_{\ell}(d)$, defined in (2.6), since this property is crucial to prove Theorem A, as observed in the final part of Sect. 2.

Lemma 4.5 Let $D>0$ be such that $y(t, D)$ has at least $\ell$ zeroes for $t \in \mathbb{R}$, so that $T_{\ell}(D)$ is well defined. Assume that $\dot{y}\left(T_{\ell}(D)\right) \neq 0$, then the functions $T_{\ell}(d)$ and $R_{\ell}(d)$, introduced in (2.6), are continuous in $d=D$.

Proof Fix $\tau<T_{\ell}(D)$. According to Remark 2.4, we set $\boldsymbol{Q}(D)=\boldsymbol{\phi}(\tau, D) \in W^{u}(\tau)$. Notice that $y\left(T_{\ell}(D) ; \tau, \boldsymbol{Q}(D)\right)=0$ and $\dot{y}\left(T_{\ell}(D) ; \tau, \boldsymbol{Q}(D)\right) \neq 0$. We assume that $\dot{y}\left(T_{\ell}(D) ; \tau, \boldsymbol{Q}(D)\right)>0$ (i.e. $\ell$ even); the case $\dot{y}\left(T_{\ell}(D) ; \tau, \boldsymbol{Q}(D)\right)<0$ ( $\ell$ odd) is analogous. Then, for every small $\Delta t \in\left(0, T_{\ell}(D)-\tau\right)$ we can find $c>0$ such that

$$
y\left(T_{\ell}(D)-\Delta t ; \tau, \boldsymbol{Q}(D)\right)<-c<0<c<y\left(T_{\ell}(D)+\Delta t ; \tau, \boldsymbol{Q}(D)\right),
$$




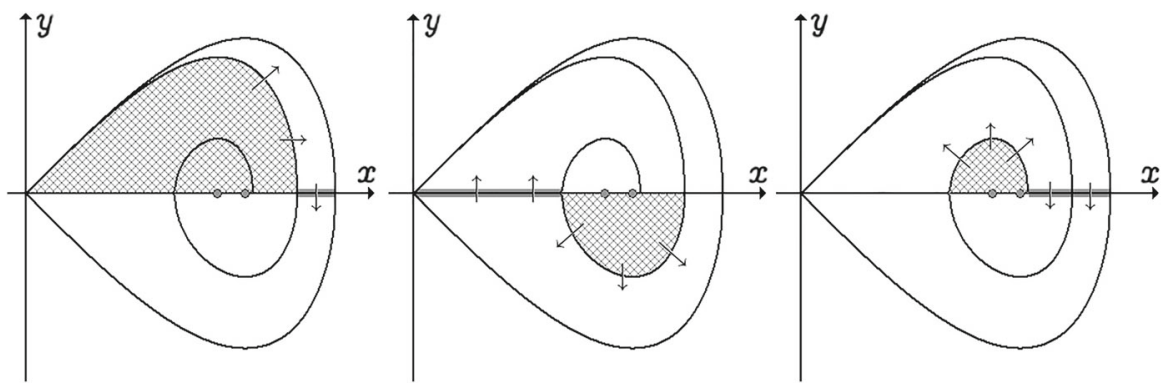

Fig. 2 The sets $F_{1}$ and $L_{1} ; F_{2}$ and $L_{2} ; F_{3}$ and $L_{3}$, respectively

and $\dot{y}(t ; \tau, Q(D))>0$ for any $t \in\left[T_{\ell}(D)-\Delta t, T_{\ell}(D)+\Delta t\right]$. Using continuous dependence on initial data, we can choose $\sigma>0$ such that $\|\boldsymbol{\phi}(t ; \tau, \boldsymbol{Q}(D))-\boldsymbol{\phi}(t ; \tau, \boldsymbol{Q})\|<c$, whenever $\|\boldsymbol{Q}-\boldsymbol{Q}(D)\|<\sigma$ for every $t \in\left[\tau, T_{\ell}(D)+\Delta t\right]$. From Remark 2.4 , for any $\sigma>0$ we can find $\delta>0$ with the following property: if $|d-D|<\delta$, then $\|\boldsymbol{Q}(d)-\boldsymbol{Q}(D)\|<\sigma$, where $\boldsymbol{Q}(d):=\boldsymbol{\phi}(\tau, d) \in W^{u}(\tau)$. Summing up, if $|d-D|<\delta$ we get

$$
y\left(T_{\ell}(D)-\Delta t ; \tau, \boldsymbol{Q}(d)\right)<0<y\left(T_{\ell}(D)+\Delta t ; \tau, \boldsymbol{Q}(d)\right)
$$

and we can assume $\dot{y}(t ; \tau, \boldsymbol{Q}(d))>0$ for any $t \in\left[T_{\ell}(D)-\Delta t, T_{\ell}(D)+\Delta t\right]$, too. Hence, $T_{\ell}(d)$ is uniquely defined and $\left|T_{\ell}(D)-T_{\ell}(d)\right|<\Delta t$ holds. This concludes the proof.

We emphasize that the transversality assumption in Lemma 4.5 is not just a technical condition: the continuity of $T_{\ell}(d)$ and $R_{\ell}(d)$ might indeed be lost removing this condition.

In order to prove the continuity of $T_{j}(d)$, we introduce some useful notation.

Let $\boldsymbol{B}_{\mathbf{0}}=\left(B_{0}, 0\right)=\Gamma_{0} \cap\{(x, 0) \mid x>0\}$, i.e. $\boldsymbol{B}_{\mathbf{0}}=\boldsymbol{A}_{\mathbf{1}}(0)$. Assume $0<\varepsilon<\varepsilon_{\ell}$; from Lemma 3.7 we can construct the sets $\mathcal{A}_{j}$ for $j \in\{1, \ldots, \ell\}$. Let $F_{j}$ be the bounded set enclosed by $\mathcal{A}_{j}$ and the line $y=0$ (see Fig. 2), i.e.

$$
\begin{aligned}
& F_{2 i+1}=\left\{(x, y) \mid H_{\varepsilon}(x, y) \leq \mathcal{H}_{2 i}, \quad y \geq 0, x \geq 0\right\}, \\
& F_{2 i+2}=\left\{(x, y) \mid H_{0}(x, y) \leq \mathcal{H}_{2 i+1}, \quad y \leq 0, \quad x \geq 0\right\} .
\end{aligned}
$$

Define

$$
\begin{aligned}
& L_{2 i+1}:=\left\{(x, 0) \mid A_{2 i+1}<x<B_{0}\right\}, \\
& L_{2 i+2}:=\left\{(x, 0) \mid 0<x<A_{2 i+2}\right\} .
\end{aligned}
$$

Following the procedure developed in the proof of Lemma 4.2, from (3.5), (3.7) and (3.17), we easily get the following result which is crucial to prove the continuity of $T_{\ell}(d)$ in its whole domain.

Lemma 4.6 Assume (1.5) with $0<\varepsilon \leq \varepsilon_{\ell}$, where $\varepsilon_{\ell}$ is the computable constant given by Lemma 3.7; then the flow of (2.2) on $\mathcal{A}_{j}$ points towards the exterior of $F_{j}$ for every $j \in$ $\{1, \ldots, \ell\}$. Moreover, the flow of (2.2) on $L_{j}$ points towards $y>0$ if $j$ is even, respectively, towards $y<0$ if $j$ is odd.

Now we are in a position to obtain the continuity of $T_{j}(d)$.

Lemma 4.7 Assume $\left(\mathbf{K}_{\mathbf{1}}\right)$ and (1.5) with $0<\varepsilon \leq \varepsilon_{\ell}$. Then, the functions $T_{j}(d), j=1, \ldots, \ell$, are continuous in their domains, provided that $T_{j}(d) \leq 0$. 
Proof Let us fix $j \in\{1 \ldots, \ell\}$. Let $d>0$ be such that $T_{j}(d)$ is well defined and $T_{j}(d) \leq 0$ holds. Then, the solution $\phi(\cdot, d)$ intersects the $x$-axis at $T_{j}(d)$ in the point $\xi_{j}\left(T_{j}(d)\right)=$ $\boldsymbol{\phi}\left(T_{j}(d), d\right)$. By Lemma 4.6, the solution $\phi(\cdot, d)$ is driven by the spiral $\boldsymbol{\gamma}$ around the points $\boldsymbol{P}^{*}(\varepsilon)$ and $\boldsymbol{P}^{*}(0)$ remaining inside the set $\Gamma_{0}$ in $(-\infty, 0)$ (cf. Lemma 3.2) and, finally, $\xi_{j}\left(T_{j}(d)\right) \in L_{j}$ with $\dot{y}\left(T_{j}(d), d\right) \neq 0$. Then, by Lemma 4.5 the continuity of $T_{j}$ in $d$ follows.

Remark 4.8 Notice that Proposition 2.10 follows from Lemma 4.7.

We state the following result, which is a translation of Proposition 2.9.

Lemma 4.9 Assume (1.5) with $0<\varepsilon \leq \varepsilon_{1}$. Then, $T_{1}(d)$ is continuous for any $d>0$ and

$$
\lim _{d \rightarrow 0} T_{1}(d)=+\infty, \quad \lim _{d \rightarrow+\infty} T_{1}(d)=-\infty,
$$

Proof Combining Lemmas 4.2 and 4.5 with the absence of invariant sets in the interior of $E_{1}$, we see that the function $T_{1}(d)$ is well defined for any $d>0$ and it is continuous. From Remark 4.3, we know that for any $\tau \in \mathbb{R}$ there is $d_{*}(\tau)>0$ such that $T_{1}\left(d_{*}(\tau)\right)=\tau$, hence $T_{1}(\cdot):(0,+\infty) \rightarrow \mathbb{R}$ is surjective. According to Remark 2.4 , we can parametrize $\tilde{W}^{u}(\tau)$ by $\tilde{Q}^{u}(d)$ so that $\tilde{Q}^{u}(0)=(0,0)$ and $\tilde{Q}^{u}\left(d^{*}(\tau)\right)=\boldsymbol{\xi}_{\mathbf{1}}(\tau)$. Hence, from Remark 4.3 it easily follows that $T_{1}(d) \rightarrow+\infty$ as $d \rightarrow 0$.

Observe now that $d^{*}(\tau) \rightarrow+\infty$ as $\tau \rightarrow-\infty$. In fact, by Remark $2.5 u^{\prime}\left(r, d^{*}(\tau)\right)<0$ for any $0<r<e^{\tau}$, which, combined with (2.1) and (3.9), leads to

$$
d^{*}(\tau)>u\left(e^{\tau}, d^{*}(\tau)\right)>P_{x}^{*}(\varepsilon) e^{-\alpha \tau} \rightarrow+\infty \quad \text { as } \tau \rightarrow-\infty .
$$

Recalling that $T_{1}\left(d^{*}(\tau)\right)=\tau$, we see that there is $d_{k} \nearrow+\infty$ such that $T_{1}\left(d_{k}\right) \rightarrow-\infty$. This is what is actually needed for the argument of this paper. However, we show that $T_{1}(d) \rightarrow-\infty$ as $d \rightarrow+\infty$.

Assume by contradiction that there are $M>0$ and $\tilde{d}_{m} \nearrow+\infty$ such that $T_{1}\left(\tilde{d}_{m}\right)>-M$ for any $m$. Then, for any $m$ we can choose $k$ such that $d_{k} \leq \tilde{d}_{m}<d_{k+1}$; we can assume without loss of generality that $T_{1}\left(d_{k}\right)<-M-1, T_{1}\left(d_{k+1}\right)<-M-1$, while $T_{1}\left(\tilde{d}_{m}\right)>-M$. Let us fix $\tau_{k}=1+\max \left\{T_{1}\left(d_{k}\right) ; T_{1}\left(d_{k+1}\right)\right\}$ and denote by $\breve{W}^{u}\left(\tau_{k}\right)$ the branch of $W^{u}\left(\tau_{k}\right)$ between the origin and $\tilde{\boldsymbol{Q}}^{\boldsymbol{u}}\left(d_{k+1}\right)$. Following $\breve{W}^{u}\left(\tau_{k}\right)$ from the origin towards $\tilde{\boldsymbol{Q}}^{\boldsymbol{u}}\left(d_{k+1}\right)$, we meet $\tilde{Q}^{u}\left(d_{k}\right)$ and then $\tilde{Q}^{u}\left(\tilde{d}_{m}\right)$. Hence, $\tilde{W}^{u}\left(\tau_{k}\right)$ enters the set $E_{1}$ defined in (4.1), it crosses the $x$ positive semi-axis until it gets to $\tilde{Q}^{u}\left(d_{k}\right)$ which lies in $y<0<x$, then it bends and gets back to $\tilde{Q}^{u}\left(\tilde{d}_{m}\right) \in E_{1}$, then it bends again and gets to $\tilde{Q}^{u}\left(d_{k+1}\right)$ which lies again in $y<0<x$. But this is in contradiction with Remark 2.2, since $\breve{W}^{u}\left(\tau_{k}\right)$ is $C^{1}$ close to the corresponding branch of $W^{u}(-\infty)$. In fact, we can find a segment transversal to $W^{u}(-\infty)$ which has a tangency point with $\breve{W}^{u}\left(\tau_{k}\right)$.

Now Theorem A easily follows from Lemmas 4.4 and 4.7.

Proof of Theorem $A$ Let us fix $\ell \geq 2,0<\varepsilon \leq \varepsilon_{\ell}$ and $j \in\{1,2, \ldots, \ell\}$. Let us define

$$
\hat{I}_{j}:=\left\{d>0 \mid T_{j}(d)<0\right\} .
$$

Obviously, $\hat{I}_{j}$ is a subset of $I_{j}$ defined in (2.5). Since $T_{j}(d)$ is continuous when it is negative (cf. Lemma 4.7), it is easy to see that $\hat{I}_{j}$ is open. Furthermore, there is $a_{1}>0$ such that $\hat{I}_{j} \subset \hat{I}_{1} \subset\left(a_{1},+\infty\right)$, cf. (4.3). From Lemma 4.4, we know that $\hat{I}_{j} \neq \emptyset$. Hence, we can find an interval $\left(a_{j}, b_{j}\right) \subset \hat{I}_{j}$ such that $a_{j} \notin \hat{I}_{j}$, and $a_{j} \geq a_{1}>0$. Notice that $b_{j}$ can be $+\infty$, and, in fact, $b_{1}=+\infty$. 
We claim that $T_{j}\left(a_{j}\right)=0$ for any $j \in\{1,2, \ldots, \ell\}$. In fact, let us consider a sequence $d^{k} \searrow a_{j}$. Since $T_{j}$ is continuous, $\lim _{k \rightarrow \infty} T_{j}\left(d^{k}\right)=T_{j}\left(a_{j}\right) \leq 0$. But, if $T_{j}\left(a_{j}\right)<0$ then $a_{j} \in \hat{I}_{j}$, which is a contradiction. Therefore, $T_{j}\left(a_{j}\right)=0$ and the claim is proved.

Then, it follows that $x\left(t, a_{j}\right)$ is positive, and $y\left(t, a_{j}\right)$ has exactly $j$ zeroes for $t \leq 0$, so Theorem A follows from Remark 2.6.

\section{Computation of $\varepsilon_{\ell}^{\prime}$ s. Proof of Theorem 1.1}

In this section, we provide a procedure in order to obtain the values $\varepsilon_{\ell}$ presented in Theorem 1.1. Let us recall that the explicit formula for $\varepsilon_{1}$, cf. (1.8) and (1.11), has been already deduced from Eq. (3.9).

We begin by giving an algorithm which allows to compute explicitly the values of $\varepsilon_{\ell}$ for Eq. (1.9) for any $\ell$ and any dimension $n$. In particular, when $\sigma=0$, i.e. (1.3) is considered, we obtain the table (1.7). However, these values are not expressed by close formulas.

Then, we obtain an explicit formula for $\varepsilon_{2}$ in Proposition 5.3, while $\varepsilon_{3}$ is given as a solution of an algebraic equation in Proposition 5.4. Finally, in Proposition 5.5 we prove the surprisingly simple formula for the $n=4$ and $\sigma=0$ case: $\varepsilon_{\ell}=1 / \ell$.

As illustrated in Sect. 3, in order to control the behaviour of the solutions of system (2.2) converging to the origin as $t \rightarrow-\infty$, we need to construct a spiral-like path $\boldsymbol{\gamma}$. This curve is built by gluing together branches of different level curves of the energy functions $H_{\varepsilon}$ and $H_{0}$ introduced in (3.4).

The first branch of $\boldsymbol{\gamma}$ is defined when $0<\varepsilon \leq \varepsilon_{1}$, and is made up by the 0 -level curve $\mathcal{A}_{1}$ of $H_{\varepsilon}$, see (3.10), which connects the origin $\boldsymbol{O}$ and the point $\boldsymbol{A}_{\mathbf{1}}(\varepsilon)=\left(A_{1}(\varepsilon), 0\right)$ defined in (3.8); we set $\mathcal{H}_{1}=H_{0}\left(\boldsymbol{A}_{1}(\varepsilon)\right)$ as in (3.11).

The second branch exists for $0<\varepsilon \leq \varepsilon_{2}$ (defined just below) and it is made up by $\mathcal{A}_{2}$ which is part of the $\mathcal{H}_{1}$-level curve of $H_{0}$, see (3.12): it connects $\boldsymbol{A}_{\mathbf{1}}(\varepsilon)$ and $\boldsymbol{A}_{\mathbf{2}}(\varepsilon)=\left(A_{2}(\varepsilon), 0\right)$. We denote by $\varepsilon_{2}>0$ the unique value such that $A_{2}\left(\varepsilon_{2}\right)=P_{x}^{*}\left(\varepsilon_{2}\right)$, so that $A_{2}(\varepsilon)<P_{x}^{*}(\varepsilon)$ iff $0<\varepsilon<\varepsilon_{2}$, and we set $\mathcal{H}_{2}=H_{\varepsilon}\left(\boldsymbol{A}_{2}\right)$. Then, the third branch exists for $0<\varepsilon \leq \varepsilon_{3}$ and it is made up by $\mathcal{A}_{3}$ which is part of the $\mathcal{H}_{2}$-level curve of $H_{\varepsilon}$, see (3.14): it connects $\boldsymbol{A}_{\mathbf{2}}(\varepsilon)$ and $\boldsymbol{A}_{3}(\varepsilon)=\left(A_{3}(\varepsilon), 0\right)$. Then, $\varepsilon_{3}>0$ is the unique value such that $A_{3}\left(\varepsilon_{3}\right)=P_{x}^{*}(0)$, so that $A_{3}(\varepsilon)>P_{x}^{*}(0)$ iff $0<\varepsilon<\varepsilon_{3}$, and we set $\mathcal{H}_{3}=H_{0}\left(\boldsymbol{A}_{3}\right)$.

So the iterative scheme to calculate the extremal $x$-coordinates $A_{i}$ is obtained via Lemma 3.7 and Remark 3.8, by setting $A_{0}=0$, and

$$
A_{2 i+1}=x_{2, \varepsilon}\left(\mathcal{H}_{2 i}\right)=x_{2,0}\left(\mathcal{H}_{2 i+1}\right), \quad A_{2 i+2}=x_{1,0}\left(\mathcal{H}_{2 i+1}\right)=x_{1, \varepsilon}\left(\mathcal{H}_{2 i+2}\right),
$$

which, combined with formula (5.5), allows us to determine $A_{i+1}$ from the previous term $A_{i}$, with a recursive procedure.

According to Lemma 3.7 and Remark 3.8, the procedure to draw $\boldsymbol{\gamma}$ for a certain value $\varepsilon$ can be summarized by the following algorithm: 


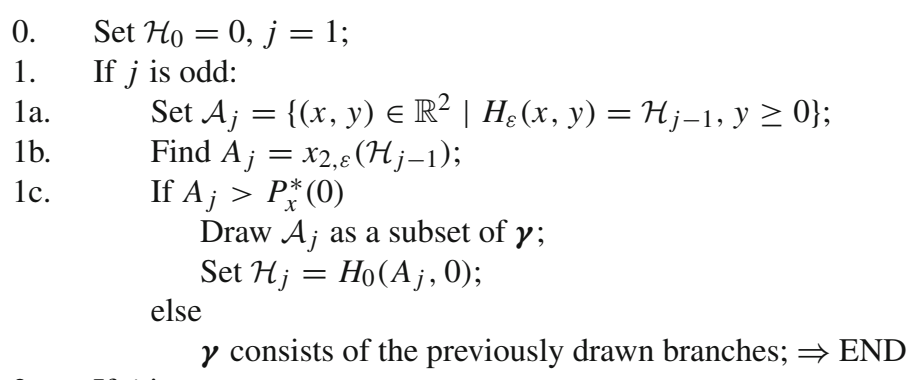

2. If $j$ is even:

2a. $\quad$ Set $\mathcal{A}_{j}=\left\{(x, y) \in \mathbb{R}^{2} \mid H_{0}(x, y)=\mathcal{H}_{j-1}, y \leq 0\right\}$;

2b. $\quad$ Find $A_{j}=x_{1,0}\left(\mathcal{H}_{j-1}\right)$;

2c. If $A_{j}<P_{x}^{*}(\varepsilon)$

Draw $\mathcal{A}_{j}$ as a subset of $\boldsymbol{\gamma}$;

else

Set $\mathcal{H}_{j}=H_{\varepsilon}\left(A_{j}, 0\right)$;

$\gamma$ consists of the previously drawn branches; $\Rightarrow$ END

3. Increase $j$ to $j+1$;

4. Go to 1 .

The critical value $\varepsilon_{\ell}$ is the only value which satisfies the identities

$$
A_{\ell}=P_{x}^{*}(0) \text { if } \ell \text { is odd, } A_{\ell}=P_{x}^{*}\left(\varepsilon_{\ell}\right) \text { if } \ell \text { is even. }
$$

Formula (5.2), combined with the explicit expression (3.7) of $P_{x}^{*}$, allows us to calculate all the values $\varepsilon_{\ell}$ with a simple shooting argument. In particular, through a rigorous computerassisted computation we obtain the table (1.7), which provides approximations from below of the values $\varepsilon_{\ell}$.

Recalling that we are treating Eq. (1.1) with $K$ as in (1.5), we can observe that the values $\varepsilon_{\ell}$ are not so small!

Remember that $G_{c}$ is defined in (3.4); furthermore $x_{1, c}(g)$ and $x_{2, c}(g)$ are the non-negative zeroes of the equation in $x, G_{c}(x)=g$ and they are, respectively, decreasing and increasing with respect to $g$, see Remark 3.5.

Let $\mathrm{R}_{c}:\left[G_{c}^{\mathrm{min}}, 0\right] \rightarrow[0,1]$ be the continuous function defined by

$$
\mathrm{R}_{c}(g)=\frac{x_{1, c}(g)}{x_{2, c}(g)} \quad \text { if } g \in\left(G_{c}^{\min }, 0\right), \quad \mathrm{R}_{c}(0)=0, \quad \mathrm{R}_{c}\left(G_{c}^{\min }\right)=1 .
$$

Remark 5.1 The function $\mathrm{R}_{c}$ is strictly decreasing.

Let us evaluate $\varepsilon_{2}$ : the stating point is (3.13), i.e. $A_{2}\left(\varepsilon_{2}\right)=P_{x}^{*}\left(\varepsilon_{2}\right)$. Then, from (3.7) and (3.8), we get

$$
A_{2}\left(\varepsilon_{2}\right)=P_{x}^{*}\left(\varepsilon_{2}\right)=\left(\frac{\alpha^{2}}{\varepsilon_{2}+1}\right)^{\frac{1}{q-2}}=A_{1}\left(\varepsilon_{2}\right)\left(\frac{2}{q}\right)^{\frac{1}{q-2}} .
$$

According to (5.3) and recalling that $A_{1}=x_{2,0}\left(\mathcal{H}_{1}\right)$ and $A_{2}=x_{1,0}\left(\mathcal{H}_{1}\right)$, the previous condition is equivalent to ask for 


$$
\Lambda:=\mathrm{R}_{0}\left(\mathcal{H}_{1}\right)=\left(\frac{2}{q}\right)^{\frac{1}{q-2}} .
$$

Definition (5.3) enables us to express $x_{1, c}$ and $x_{2, c}$ as functions of $\mathrm{R}_{c}$.

Lemma 5.2 Consider $\mathrm{R} \in(0,1)$ and $g \in\left(G_{c}^{\mathrm{min}}, 0\right)$ such that $\mathrm{R}_{c}(g)=\mathrm{R}$. Then, we get

$$
x_{2, c}(g)=\left(\frac{\alpha^{2} q}{2(c+1)} \cdot \frac{1-\mathrm{R}^{2}}{1-\mathrm{R}^{q}}\right)^{\frac{1}{q-2}}, \quad x_{1, c}(g)=\mathrm{R} x_{2, c}(g) .
$$

Proof From $G_{c}\left(x_{1, c}(g)\right)=G_{c}\left(x_{2, c}(g)\right)=g$, we deduce that

$$
\frac{c+1}{q}\left(\left[x_{2, c}(g)\right]^{q}-\left[x_{1, c}(g)\right]^{q}\right)=\frac{\alpha^{2}}{2}\left(\left[x_{2, c}(g)\right]^{2}-\left[x_{1, c}(g)\right]^{2}\right) .
$$

Then, substituting $x_{1, c}(g)=\mathrm{R} x_{2, c}(g)$, we easily complete the proof.

We are now in the position to calculate explicitly the critical value $\varepsilon_{2}$, proving (1.12) and its restriction (1.8) to the $\sigma=0$ case.

Proposition 5.3 The critical value $\varepsilon_{2}$ for (1.9) is given by the following formula

$$
\varepsilon_{2}=\frac{q-2}{q}\left[\left(\frac{q}{2}\right)^{\frac{2}{q-2}}-1\right]^{-1}
$$

which equals $\varepsilon_{2}=\frac{2}{n}\left[\left(\frac{n}{n-2}\right)^{\frac{n-2}{2}}-1\right]^{-1}$ for (1.3), i.e. when $\sigma=0$ and $q=\frac{2 n}{n-2}$.

Proof From (3.8) and Lemma 5.2 with $\mathrm{R}=\Lambda$ and $c=0$, we obtain

$$
\left(\frac{\alpha^{2} q}{2\left(\varepsilon_{2}+1\right)}\right)^{\frac{1}{q-2}}=A_{1}\left(\varepsilon_{2}\right)=\left(\frac{\alpha^{2} q}{2} \cdot \frac{1-\Lambda^{2}}{1-\Lambda^{q}}\right)^{\frac{1}{q-2}}
$$

whence

$$
\frac{1}{\varepsilon_{2}+1}=\frac{1-\Lambda^{2}}{1-\Lambda^{q}}
$$

From (5.4) and (5.7), since $\Lambda^{q-2}=\frac{2}{q}$, we get

$$
\varepsilon_{2}=\frac{1-\Lambda^{q}}{1-\Lambda^{2}}-1=\frac{\Lambda^{2}\left(\Lambda^{q-2}-1\right)}{\Lambda^{2}-1}=\frac{q-2}{q}\left[\left(\frac{q}{2}\right)^{\frac{2}{q-2}}-1\right]^{-1} .
$$

Let us now proceed with the estimate of $\varepsilon_{3}$, starting from $A_{3}\left(\varepsilon_{3}\right)=P_{x}^{*}(0)$.

Proposition 5.4 The critical value $\varepsilon_{3}$ is the unique solution of the following equation:

$$
\begin{gathered}
{\left[\mathcal{X}^{q}\left(\varepsilon_{3}\right)+\mathcal{W}\left(\varepsilon_{3}\right)\right]^{\frac{2}{q}}=\mathcal{X}^{2}\left(\varepsilon_{3}\right)+\frac{2}{q} \mathcal{W}\left(\varepsilon_{3}\right),} \\
\text { where } \mathcal{X}\left(\varepsilon_{3}\right)=\left(\frac{q}{2\left(\varepsilon_{3}+1\right)}\right)^{\frac{1}{q-2}}, \quad \mathcal{W}\left(\varepsilon_{3}\right)=1+\frac{1}{\varepsilon_{3}}\left(1-\frac{q}{2}\right),
\end{gathered}
$$

provided that $\mathcal{X}^{q}\left(\varepsilon_{3}\right)+\mathcal{W}\left(\varepsilon_{3}\right)>0$. 
Proof From (3.16) we have $G_{\varepsilon}\left(A_{3}\right)=\mathcal{H}_{2}=-\frac{\varepsilon}{q}\left(A_{1}^{q}-A_{2}^{q}\right)$. Thus, according to (3.4), we immediately infer that

$$
A_{2}^{q}=A_{1}^{q}+\frac{1+\varepsilon}{\varepsilon} A_{3}^{q}-\frac{q \alpha^{2}}{2 \varepsilon} A_{3}^{2} .
$$

Similarly, from (3.16) we find $G_{0}\left(A_{2}\right)=\mathcal{H}_{1}=-\frac{\varepsilon}{q} A_{1}^{q}$, hence

$$
A_{2}^{2}=\frac{2}{q \alpha^{2}}\left(A_{2}^{q}+\varepsilon A_{1}^{q}\right), \quad A_{2}>0 .
$$

Substituting the expression of $A_{2}$ given by (5.10) into (5.11), we obtain

$$
\left[A_{1}^{q}+\frac{1+\varepsilon}{\varepsilon} A_{3}^{q}-\frac{q \alpha^{2}}{2 \varepsilon} A_{3}^{2}\right]^{\frac{2}{q}}=\frac{2}{q \alpha^{2}}\left[(\varepsilon+1)\left(A_{1}^{q}+\frac{1}{\varepsilon} A_{3}^{q}\right)-\frac{q \alpha^{2}}{2 \varepsilon} A_{3}^{2}\right] .
$$

Since $A_{3}=A_{3}\left(\varepsilon_{3}\right)=P_{x}(0)$, see (3.7), and $A_{1}$ is given by (3.8) we get

$$
\begin{aligned}
& {\left[\left(\frac{q}{2\left(\varepsilon_{3}+1\right)}\right)^{\frac{q}{q-2}}+1+\frac{1}{\varepsilon_{3}}\left(1-\frac{q}{2}\right)\right]^{\frac{2}{q}}} \\
& =\frac{2}{q}\left[\left(\varepsilon_{3}+1\right)\left(\frac{q}{2\left(\varepsilon_{3}+1\right)}\right)^{\frac{q}{q-2}}+1+\frac{1}{\varepsilon_{3}}\left(1-\frac{q}{2}\right)\right],
\end{aligned}
$$

which coincides with Eq. (5.8). The uniqueness of the solution of this equation is guaranteed provided that $A_{2}\left(\varepsilon_{3}\right)>0$, which is equivalent to $\mathcal{X}^{q}\left(\varepsilon_{3}\right)+\mathcal{W}\left(\varepsilon_{3}\right)>0$.

Proposition 5.5 Consider (1.3), i.e. (1.9) with $\sigma=0$, and set $n=4$. Then, $\varepsilon_{\ell}=\frac{1}{\ell}$ for any $\ell \geq 1$.

Proof According to (2.1) and (3.1), we know that $\alpha=1, q=4$. Consequently, (5.5) becomes

$$
x_{1, c}(g)^{2}+x_{2, c}(g)^{2}=\frac{2}{c+1} .
$$

Hence, from (5.1) we obtain the following identities

$$
A_{2 i+1}^{2}+A_{2 i+2}^{2}=2, \quad A_{2 i}^{2}+A_{2 i+1}^{2}=\frac{2}{\varepsilon+1} .
$$

Starting from $A_{0}=0$, we can prove by induction that

$$
A_{2 i}=\sqrt{\frac{2 \varepsilon i}{1+\varepsilon}}, \quad A_{2 i+1}=\sqrt{\frac{2(1-\varepsilon i)}{1+\varepsilon}} .
$$

Combining (5.2) with (3.7), we note that if $\ell=2 \hat{i}+1$ is odd,

$$
A_{\ell}=A_{2 \hat{i}+1}=\sqrt{\frac{2\left(1-\varepsilon_{\ell} \hat{i}\right)}{1+\varepsilon_{\ell}}}=\sqrt{\frac{2-(\ell-1) \varepsilon_{\ell}}{1+\varepsilon_{\ell}}}=1=P_{x}^{*}(0),
$$

whence it follows $\varepsilon_{\ell}=\frac{1}{\ell}$. Conversely, if $\ell=2 \hat{i}$ is even, one has

$$
A_{\ell}=A_{2 \hat{i}}=\sqrt{\frac{2 \varepsilon_{\ell} \hat{i}}{1+\varepsilon_{\ell}}}=\sqrt{\frac{\ell \varepsilon_{\ell}}{1+\varepsilon_{\ell}}}=\sqrt{\frac{1}{1+\varepsilon_{\ell}}}=P_{x}^{*}\left(\varepsilon_{\ell}\right),
$$

which implies $\varepsilon_{\ell}=\frac{1}{\ell}$. This completes the proof. 
Acknowledgements F. Dalbono would like to express her gratitude to the "Centro de Matemática, Aplicações Fundamentais e Investigação Operacional" of the University of Lisbon for its hospitality. M. Franca wishes to honour prof. R. Johnson who recently passed away, for his generosity and careful guide. He was the one who introduced the author to the study of this subject and to research in mathematics.

\section{A Proof of Lemma 4.4}

In this Appendix, we reprove Lemma 4.4, and so Proposition 2.8, in order to be more selfcontained. We recall that this result has been already proved in [10, Theorem 1.6] by Chen and Lin and restated in [9, Lemma 2.2]. However, their clever proof is far from being trivial, and the argument is even more difficult to be read due to the presence of some misprints. For this reason and for completeness, we reprove it here in a slightly more general version, following the outline of the original idea, but performing some changes in certain points to make it more coherent with the dynamical ideas of the present article.

In this Appendix, we consider equation

$$
\left(u^{\prime} r^{n-1}\right)^{\prime}+r^{n-1+\sigma} K(|x|) u^{q(\sigma)-1}=0, \quad \text { where } q(\sigma)=2 \frac{n+\sigma}{n-2},
$$

and $K$ is a positive $C^{1}$ function. Our aim consists in proving the following result.

Proposition A.1 Consider Eq. (A.1), and assume both $\left(\mathbf{K}_{\mathbf{1}}\right)$ and $\left(\mathbf{K}_{\mathbf{2}}\right)$. Then, for any fixed $\ell \in \mathbb{N}$, and for any $\rho>0$ there is $d_{\ell} \in I_{\ell}$ such that $R_{\ell}\left(d_{\ell}\right)<\rho$.

Taking into account the standard rescaling argument exhibited to prove Remark 1.3, without loss of generality, from now on we will restrict ourselves to the case

$$
K(r)=1+\chi k(r), \quad 0 \leq k(r) \leq 1, \quad \forall r \in[0,1],
$$

where $\chi>0$ is a fixed constant, which need not be small. In particular, Eq. (A.1) reduces to Eq. (1.9).

Let $u(r)=u(r ; d)$ be a solution of (1.9), and let $\phi(t, d)=\phi(t)=(x(t), y(t))$ be the corresponding trajectory of (2.2). According to (3.2), $\mathcal{H}(\phi(t), t)$ is decreasing for $t \leq 0$, and, consequently, as in Lemma 3.1, $\mathcal{H}(\boldsymbol{\phi}(t), t)$ is non-positive for $t \leq 0$, and $u(r)$ and $x(t)$ are positive for $r \leq 1$ and $t \leq 0$, respectively. From the monotonicity assumption on $K$, we immediately observe that $1 \leq \mathcal{K}(t) \leq 1+\chi$, for $t \leq 0$. Thus, according to Lemma 3.2, we deduce that $\phi(t)$ belongs to the region enclosed by $\Gamma_{0}$ for $t \leq 0$, and, consequently, $\phi(t, d)$ is bounded for $t \leq 0$, uniformly in $d$; in fact $0<x(t) \leq A_{1}(0)$, see (3.8).

Furthermore, from (2.2), it is easy to check that $\dot{y}(t)>0$ as long as $0<x(t)<P_{x}^{*}(\chi) \leq$ $P_{x}^{*}\left(\chi k\left(e^{t}\right)\right)$ for $t \leq 0$, see (3.7). Let us choose $\zeta_{0}=P_{x}^{*}(\chi) / 2>0$.

Lemma A.2 Fix $\zeta<\zeta_{0}$; there exist the sequences $d^{i}, \bar{T}_{1}^{i}$ and $\bar{T}_{2}^{i}$ with $\bar{T}_{1}^{i}<\bar{T}_{2}^{i}$, d $d^{i} \nearrow+\infty$, $\bar{T}_{2}^{i} \searrow-\infty$ such that the trajectory $\boldsymbol{\phi}^{i}(t)=\left(x^{i}(t), y^{i}(t)\right)$ of $(2.2)$ corresponding to $u^{i}(r)=$ $u\left(r ; d^{i}\right)$ satisfies the following property: $x^{i}(t)<\zeta$ for $t<\bar{T}_{1}^{i}, x^{i}\left(\bar{T}_{1}^{i}\right)=\zeta, x^{i}(t)>\zeta$ for $\bar{T}_{1}^{i}<t<\bar{T}_{2}^{i}$, and $x^{i}\left(\bar{T}_{2}^{i}\right)=\zeta$.

Proof To prove this lemma, we use an argument different from [10, Theorem 1.6]. Let us observe that the level curve $\Gamma_{\chi}$ defined in (3.6) intersects the line $x=\zeta$ in two points $Q_{ \pm}^{\infty}=\left(\zeta, \pm Y^{\infty}\right)$, where $Y^{\infty}>0$. Let

$$
L:=\left\{(\zeta,-Y) \mid Y^{\infty} / 2<Y<2 Y^{\infty}\right\},
$$


and notice that the flow of the autonomous system (2.2), where $\mathcal{K}(t) \equiv K(0)=1+\chi$ is transversal on $L$, since $\zeta<\zeta_{0}$. Furthermore, $\Gamma_{\chi}$ is the graph of a homoclinic trajectory $\psi_{\tau}(t)$ of such a system, and for any $\tau \in \mathbb{R}$ we may assume that $\psi_{\tau}(\tau)=Q_{-}^{\infty}$, due to the $t$-translation invariance of the autonomous system. From Remark 2.2, we see that there is $\tau^{*}$ such that the unstable leaf $W^{u}(\tau)$ of the original non-autonomous system (2.2) intersects the line $L$ in a point $\boldsymbol{Q}(\tau)$ for any $\tau \leq \tau^{*}$. Furthermore, $\boldsymbol{Q}(\tau) \rightarrow \boldsymbol{Q}_{-}^{\infty}$ as $\tau \rightarrow-\infty$.

Let us consider the trajectory $\psi_{\tau}(t)=\bar{\phi}\left(t ; \tau, Q_{-}^{\infty}\right)$ of the frozen autonomous system (2.2) where $\mathcal{K}(t) \equiv 1+\chi$ for $\tau \leq \tau^{*}$, and the corresponding regular solution $\bar{u}(r ; \bar{d}(\tau))$ of (A.1), with $K(r) \equiv 1+\chi$. Similarly, let $\phi(t ; \tau, \boldsymbol{Q}(\tau))$ be a trajectory of the original non-autonomous system (2.2), and let $u(r ; d(\tau))$ be the corresponding regular solution of (A.1) with the original $K(r)$. Using continuous dependence on parameters, we see that $\boldsymbol{\phi}(t ; \tau, \boldsymbol{Q}(\tau))$ is close to $\boldsymbol{\psi}_{\tau}(t)$ if $t \leq \tau$ and $\tau \leq \tau^{*}$, with a possibly larger $\left|\tau^{*}\right|$. According to [20, Remark 2.5], we notice that $\bar{d}(\tau) \rightarrow+\infty$ as $\tau \rightarrow-\infty$, which implies that $d(\tau) \rightarrow+\infty$ as $\tau \rightarrow-\infty$, see also the proof of Lemma 4.9. The claim immediately follows by extracting the sequence $d^{i}=d\left(\bar{T}_{2}^{i}\right)$ which satisfies the required monotonicity properties.

Fix $\zeta<\zeta_{0}$ so that the existence of the sequences $d^{i}, \bar{T}_{1}^{i}, \bar{T}_{2}^{i}$ is guaranteed by Lemma A.2. Let $u^{i}(r)=u\left(r ; d^{i}\right)$, and let $\phi^{i}(t)=\left(x^{i}(t), y^{i}(t)\right)$ be the corresponding trajectory of (2.2).

Lemma A.3 For any $M>0$, there are $r_{0}$ and $i_{0}$ such that $u^{i}\left(r_{0}\right) \geq M$ for any $i \geq i_{0}$.

Proof From now on, we follow quite closely the ideas of [10]. Assume, by contradiction, that the lemma is false; then there exists $M>0$ with the following property: for any $r_{0}>0$, there is a subsequence, still denoted by $u^{i}$, such that $u^{i}\left(r_{0}\right)<M$ for any $i$.

Fix $\delta>0$ small enough to satisfy

$$
\beta=2\left(\frac{\alpha}{l}-1\right)-\frac{\delta}{\alpha-\delta}>0 \quad \text { and } \quad \delta<\alpha-l,
$$

where the constant $l \in(0, \alpha)$ is given by assumption $\left(\mathbf{K}_{\mathbf{2}}\right)$. We set $\tilde{\zeta}=\tilde{\zeta}(\delta)=$ $\min \left[\left(\frac{2 \delta \alpha-\delta^{2}}{K(0)}\right)^{\frac{1}{q-2}} ; \zeta\right]$, so that from (2.2) we find

$$
\ddot{x}^{i}(t)=\dot{y}^{i}(t)>(\alpha-\delta)^{2} x^{i}(t), \quad \text { for any } 0<x<\tilde{\zeta}, \quad t \leq 0 .
$$

Taking into account $\left(\mathbf{K}_{2}\right)$, we choose $r_{0}$ so small that $M r_{0}^{(n-2) / 2}<\tilde{\zeta} / 2$, and

$$
-3 \frac{l A}{2} r^{l-1} \leq K^{\prime}(r) \leq-\frac{l A}{2} r^{l-1}, \quad \forall r \in\left(0, r_{0}\right)
$$

Set $T_{0}=\ln \left(r_{0}\right)<0$. Recalling that $\bar{T}_{2}^{i} \searrow-\infty$, we can find $i_{0} \in \mathbb{N}$ such that $\bar{T}_{1}^{i}<\bar{T}_{2}^{i}<T_{0}$ for any $i \geq i_{0}$. Without loss of generality, we now restrict ourselves to consider the sequence $u^{i}$ with $i \geq i_{0}$. Thus,

$$
x^{i}\left(T_{0}\right)=u^{i}\left(r_{0}\right) r_{0}^{(n-2) / 2}<M r_{0}^{(n-2) / 2}<\tilde{\zeta} / 2 .
$$

According to Lemma A.2, we define

$$
\bar{T}^{i}=\max \left\{t \in\left[\bar{T}_{2}^{i} ; T_{0}\right) \mid x^{i}(t) \geq \tilde{\zeta}\right\},
$$

so that $x^{i}(t)<\tilde{\zeta}$ when $\bar{T}^{i}<t<T_{0}$, hence (A.4) holds and $\ddot{x}^{i}(t)>0$ in this interval. So, we have two cases: either $\dot{x}^{i}(t)<0$ for any $\bar{T}^{i}<t<T_{0}$, or $x^{i}(t)$ has a local minimum at $t=\mathcal{T} \in\left(\bar{T}^{i}, T_{0}\right)$. 
Case 1) Assume $\dot{x}^{i}(t)<0$ for any $\bar{T}^{i}<t<T_{0}$.

Let $E_{\delta}(x, y)=y^{2}-(\alpha-\delta)^{2} x^{2}$. According to (2.2), we deduce that

$$
\dot{E}_{\delta}\left(x^{i}(t), y^{i}(t)\right)=2 \dot{x}^{i}(t)\left(\dot{y}^{i}(t)-(\alpha-\delta)^{2} x^{i}(t)\right) .
$$

From (A.4), we see that $E_{\delta}\left(x^{i}(t), y^{i}(t)\right)$ is strictly decreasing if $\bar{T}^{i}<t<T_{0}$. Hence, $E_{\delta}\left(\phi^{i}(t)\right)>E_{\delta}\left(\phi^{i}\left(T_{0}\right)\right)$ for any $\bar{T}^{i}<t<T_{0}$, so that from (2.2) we find

$$
\left[\dot{x}^{i}(t)\right]^{2}=\left[y^{i}(t)\right]^{2}>(\alpha-\delta)^{2}\left[\left(x^{i}(t)\right)^{2}-\left(x^{i}\left(T_{0}\right)\right)^{2}\right] .
$$

Since $x^{i}(t)$ is decreasing, the right hand side of (A.9) is positive in $\left(\bar{T}^{i}, T_{0}\right)$, hence

$$
\frac{-\dot{x}^{i}(t)}{(\alpha-\delta) \sqrt{\left(x^{i}(t)\right)^{2}-\left(x^{i}\left(T_{0}\right)\right)^{2}}}>1
$$

for any $\bar{T}^{i}<t<T_{0}$. Integrating, we get

$$
\begin{aligned}
T_{0}-\bar{T}^{i} & <\frac{1}{\alpha-\delta} \int_{x^{i}\left(\bar{T}^{i}\right)}^{x^{i}\left(T_{0}\right)} \frac{-\mathrm{d} x}{\sqrt{x^{2}-\left[x^{i}\left(T_{0}\right)\right]^{2}}}=\frac{1}{\alpha-\delta} \int_{1}^{\tilde{\zeta} / x^{i}\left(T_{0}\right)} \frac{\mathrm{d} z}{\sqrt{z^{2}-1}} \\
& =\frac{1}{\alpha-\delta}\left[\ln \left(z+\sqrt{z^{2}-1}\right)\right]_{1}^{\tilde{\zeta} / x^{i}\left(T_{0}\right)} \leq \frac{1}{\alpha-\delta} \ln \left(\frac{2 \tilde{\zeta}}{x^{i}\left(T_{0}\right)}\right) .
\end{aligned}
$$

At this point, we need assumption $\left(\mathbf{K}_{2}\right)$ to estimate $\bar{T}^{i}$ from above. Since $x^{i}\left(\bar{T}^{i}\right)=\tilde{\zeta}$, cf. (A.7), and $x^{i}\left(T_{0}\right)<\tilde{\zeta} / 2$, cf. (A.6), there is $\tilde{T} \in\left(\bar{T}^{i}, T_{0}\right)$ such that $x^{i}(\tilde{T})=\tilde{\zeta} / 2$. Furthermore, $y^{i}(t)$ is bounded, so there is $c>0$ such that $\tilde{T}-\bar{T}^{i}>c \tilde{\zeta}$. Actually, it might be shown that $\tilde{T}-\bar{T}^{i}>\ln (2) / \alpha$ as a consequence of the negativity of $\mathcal{H}\left(\phi^{i}(t), t\right)$ for $t \leq 0$ and using some Gronwall estimates. Thus, from (3.1) and (3.2) we find

$$
-\frac{\alpha^{2}}{2}\left[x^{i}\left(T_{0}\right)\right]^{2} \leq \mathcal{H}\left(\boldsymbol{\phi}^{i}\left(T_{0}\right), T_{0}\right)=\mathcal{H}\left(\boldsymbol{\phi}^{i}\left(\bar{T}^{i}\right), \bar{T}^{i}\right)+\int_{\bar{T}^{i}}^{T_{0}} \dot{\mathcal{K}}(t) \frac{\left[x^{i}(t)\right]^{q}}{q} \mathrm{~d} t .
$$

Then, recalling that $\mathcal{H}\left(\phi^{i}(t), t\right)$ and $\dot{\mathcal{K}}(t)$ are non-positive and $x^{i}(t)$ decreases when $t \in$ $\left[\bar{T}^{i}, T_{0}\right]$, from $\left(\mathbf{K}_{2}\right)$ and (A.5), we obtain

$$
\begin{aligned}
\left|x^{i}\left(T_{0}\right)\right|^{2} & \geq \frac{2}{\alpha^{2}} \int_{\bar{T}^{i}}^{\tilde{T}}|\dot{\mathcal{K}}(t)| \frac{\left|x^{i}(t)\right|^{q}}{q} \mathrm{~d} t \geq \frac{\tilde{\zeta}^{q}}{q \alpha^{2} 2^{q-1}} \int_{\bar{T}^{i}}^{\tilde{T}}|\dot{\mathcal{K}}(t)| \mathrm{d} t \\
& \geq \frac{l A}{q \alpha^{2} 2^{q}} \tilde{\zeta}^{q} e^{l \bar{T}^{i}}\left(\tilde{T}-\bar{T}^{i}\right)>c_{1}^{2} e^{l \bar{T}^{i}} \tilde{\zeta}^{q+1}
\end{aligned}
$$

where $c_{1}=\frac{1}{\alpha} \sqrt{\frac{l A c}{q 2^{q}}}$. Hence, by (A.6)

$$
M e^{\alpha T_{0}}>u^{i}\left(r_{0}\right) e^{\alpha T_{0}}=x^{i}\left(T_{0}\right)>c_{1} e^{\frac{l \bar{T}^{i}}{2}} \tilde{\zeta}^{\frac{q+1}{2}},
$$

which implies

$$
\frac{l}{2} \bar{T}^{i}<\alpha T_{0}+\ln \left(\frac{M}{c_{1}}\right)-\frac{q+1}{2} \ln (\tilde{\zeta}) .
$$

Plugging the second inequality of (A.14) in (A.11), we get

$$
T_{0}<\frac{(q-1)}{2(\alpha-\delta) \ln \left(\frac{1}{\tilde{\zeta}}\right)}+\left(1-\frac{l}{2(\alpha-\delta)}\right) \bar{T}^{i}+c_{2},
$$


where $c_{2}=\frac{\ln (2)-\ln \left(c_{1}\right)}{\alpha-\delta}$. Then, from (A.16) and (A.15) we find

$$
\begin{aligned}
T_{0}< & \frac{(q-1)}{2(\alpha-\delta)} \ln \left(\frac{1}{\tilde{\zeta}}\right)+ \\
& +\frac{2}{l}\left(1-\frac{l}{2(\alpha-\delta)}\right)\left[\alpha T_{0}+\ln \left(\frac{M}{c_{1}}\right)+\frac{q+1}{2} \ln \left(\frac{1}{\tilde{\zeta}}\right)\right]+c_{2} .
\end{aligned}
$$

From the choice of $\delta$ in (A.3), $\frac{2 \alpha}{l}\left(1-\frac{l}{2(\alpha-\delta)}\right)-1=2\left(\frac{\alpha}{l}-1\right)-\frac{\delta}{\alpha-\delta}=\beta>0$, then there are $c_{3}>0, c_{4}>0$ such that (A.17) can be written as follows

$$
-\beta T_{0}<c_{3} \ln \left(\frac{1}{\tilde{\zeta}}\right)+c_{4} \ln \left(\frac{M}{c_{1}}\right)+c_{2} .
$$

Since $\tilde{\zeta}>0$ and $M>0$ are fixed, we can let $T_{0}$ go to $-\infty$, obtaining a contradiction with (A.18), and the lemma in Case $\mathbf{1}$ is proved.

Case 2) Assume that there is $\mathcal{T} \in\left(\bar{T}^{i}, T_{0}\right)$ such that $\dot{x}^{i}(t)<0$ for $\bar{T}^{i} \leq t<\mathcal{T}$ and $\dot{x}^{i}(t)>0$ for $\mathcal{T}<t \leq T_{0}$.

Repeating the argument of Case 1 for $\bar{T}^{i} \leq t<\mathcal{T}$, we go through (A.9) and (A.10) and we find

$$
\mathcal{T}-\bar{T}^{i}<\frac{1}{\alpha-\delta} \ln \left(\frac{2 \tilde{\zeta}}{x^{i}(\mathcal{T})}\right) .
$$

Now we estimate $T_{0}-\mathcal{T}$ with analogous techniques. In particular, from (A.4) and (A.8) we see that $E_{\delta}\left(x^{i}(t), y^{i}(t)\right)$ is strictly increasing if $\mathcal{T}<t<T_{0}$. Hence, $E_{\delta}\left(\phi^{i}(t)\right)>E_{\delta}\left(\phi^{i}(\mathcal{T})\right)$ for any $\mathcal{T}<t<T_{0}$, and, consequently,

$$
\left[\dot{x}^{i}(t)\right]^{2}=\left[y^{i}(t)\right]^{2}>(\alpha-\delta)^{2}\left[\left(x^{i}(t)\right)^{2}-\left(x^{i}(\mathcal{T})\right)^{2}\right] .
$$

Since $\dot{x}^{i}(t)>0$, the right hand side of (A.20) is positive in $\left(\mathcal{T}, T_{0}\right)$, hence

$$
\frac{\dot{x}^{i}(t)}{(\alpha-\delta) \sqrt{\left(x^{i}(t)\right)^{2}-\left(x^{i}(\mathcal{T})\right)^{2}}}>1, \quad \forall t \in\left(\mathcal{T}, T_{0}\right) .
$$

Integrating, we get

$$
\begin{aligned}
T_{0}-\mathcal{T} & <\frac{1}{\alpha-\delta} \int_{x^{i}(\mathcal{T})}^{x^{i}\left(T_{0}\right)} \frac{\mathrm{d} x}{\sqrt{x^{2}-\left[x^{i}(\mathcal{T})\right]^{2}}}=\frac{1}{\alpha-\delta} \int_{1}^{x^{i}\left(T_{0}\right) / x^{i}(\mathcal{T})} \frac{\mathrm{d} z}{\sqrt{z^{2}-1}}= \\
& =\frac{1}{\alpha-\delta}\left[\ln \left(z+\sqrt{\left.z^{2}-1\right)}\right]_{1}^{x^{i}\left(T_{0}\right) / x^{i}(\mathcal{T})} \leq \frac{1}{\alpha-\delta} \ln \left(\frac{2 x^{i}\left(T_{0}\right)}{x^{i}(\mathcal{T})}\right) .\right.
\end{aligned}
$$

Combining (A.19) with (A.21), we conclude that

$$
T_{0}-\bar{T}^{i}<\frac{1}{\alpha-\delta} \ln \left(\frac{2 \tilde{\zeta}}{x^{i}(\mathcal{T})}\right)+\frac{1}{\alpha-\delta} \ln \left(\frac{2 x^{i}\left(T_{0}\right)}{x^{i}(\mathcal{T})}\right) .
$$

Moreover, notice that (A.12) and (A.13) keep on holding by replacing $T_{0}$ with $\mathcal{T}$. In particular, according to (A.6), the following inequalities hold:

$$
x^{i}(\mathcal{T})>c_{1} e^{\frac{l \bar{T}^{i}}{2}} \tilde{\zeta} \frac{q+1}{2}, \quad x^{i}\left(T_{0}\right)<M e^{\alpha T_{0}},
$$

which, plugged in (A.22), lead to

$$
T_{0}-\bar{T}^{i}<\frac{\alpha T_{0}}{\alpha-\delta}-\frac{l \bar{T}^{i}}{\alpha-\delta}+\frac{q}{\alpha-\delta} \ln \left(\frac{1}{\tilde{\zeta}}\right)+\frac{\ln M}{\alpha-\delta}+2 c_{2} .
$$


Hence, there are $\tilde{c}_{3}>0$ and $\tilde{c}_{4}>0$ such that

$$
\left(-1+\frac{l}{\alpha-\delta}\right) \bar{T}^{i}<\frac{\delta}{\alpha-\delta} T_{0}+\tilde{c}_{3} \ln \left(\frac{1}{\tilde{\zeta}}\right)+\tilde{c}_{4} \ln M+2 c_{2} .
$$

Taking into account that $T_{0}<0$, we finally infer that

$$
\left(-1+\frac{l}{\alpha-\delta}\right) \bar{T}^{i}<\tilde{c}_{3} \ln \left(\frac{1}{\tilde{\zeta}}\right)+\tilde{c}_{4} \ln M+2 c_{2},
$$

where $\left(-1+\frac{l}{\alpha-\delta}\right)<0$ by (A.3). Since $\tilde{\zeta}>0$ and $M>0$ are fixed, while $\bar{T}^{i}<T_{0}$ is arbitrarily small, we can let $T_{0}$ and, consequently, $\bar{T}^{i}$ go to $-\infty$, obtaining a contradiction with (A.23). This proves Case 2.

Now we are ready to state the result proved in [10, Theorem 1.6] from which Proposition A.1 and Lemma 4.4 follow.

Proposition A.4 Consider Eq. (A.1) under the condition (A.2), and assume both (K. $\mathbf{K}_{\mathbf{1}}$ ) and $\left(\mathbf{K}_{2}\right)$. Then, there is a singular solution $u^{\infty}(r)$ such that $\liminf _{r \rightarrow 0} u^{\infty}(r) r^{\alpha}=0$ and $\limsup _{r \rightarrow 0} u^{\infty}(r) r^{\alpha}=A_{1}(\chi)$.

Proof Since $u^{i}(r)<A_{1}(0) r^{-\alpha}$ and there is $c>0$ such that $\left|u^{\prime i}(r)\right|<c r^{-(\alpha+1)}$ for any $0 \leq r<1$, it follows that, up to subsequences, $u^{i}(r)$ converges uniformly in any compact interval of $(0,1]$ to a function, say $u^{\infty}(r)$, together with its derivative. Notice that $u^{\infty}(r)$ is a solution of (A.1); furthermore, from Lemma A.3 we know that $\lim _{r \rightarrow 0} u^{\infty}(r)=+\infty$, so $u^{\infty}$ is singular. Let $\boldsymbol{\phi}^{\infty}(t)=\left(x^{\infty}(t), y^{\infty}(t)\right)$ be the trajectory of (2.2) corresponding to $u^{\infty}(r)$.

Using (3.2) and Lebesgue convergence theorem for any $t \leq 0$, we find

$$
\begin{aligned}
\mathcal{H}\left(\boldsymbol{\phi}^{\infty}(t), t\right) & =\lim _{i \rightarrow+\infty} \mathcal{H}\left(\boldsymbol{\phi}^{i}(t), t\right) \\
& =\lim _{i \rightarrow+\infty} \int_{-\infty}^{t} \dot{\mathcal{K}}(s) \frac{\left[x^{i}(s)\right]^{q}}{q} \mathrm{~d} s=\int_{-\infty}^{t} \dot{\mathcal{K}}(s) \frac{\left[x^{\infty}(s)\right]^{q}}{q} \mathrm{~d} s .
\end{aligned}
$$

Since $\dot{\mathcal{K}}(t) \frac{\left[x^{\infty}(t)\right]^{q}}{q} \in L^{1}((-\infty, 0])$, we see that $\lim _{t \rightarrow-\infty} \mathcal{H}\left(\boldsymbol{\phi}^{\infty}(t), t\right)=0$. Being $u^{\infty}(r) \mathrm{a}$ singular solution, we conclude that $x^{\infty}(t) \nrightarrow(0,0)$ as $t \rightarrow-\infty$, so $x^{\infty}$ has the whole $\Gamma_{\chi}$ as $\alpha$-limit set, and the proposition follows.

Proof of Proposition A.1 Proposition A.1 now follows immediately from Proposition A.4 recalling that $u^{i}(r)=u\left(r ; d^{i}\right) \rightarrow u^{\infty}(r)$ uniformly in any compact subset of $(0,1]$. For any fixed $\rho>0$ and $\ell \in \mathbb{N}$ we can find $\rho_{\ell}<\rho$ such that $\phi^{\infty}(t)$ intersects the $x$-axis at least $\ell$ times in the interval $\left(\ln \rho_{\ell}, \ln \rho\right)$. So, the same property holds for $\phi^{i}(t)$, when the index $i$ is sufficiently large; hence, for any $\ell$ we find $\phi^{i_{\ell}}(t)=\phi\left(t, d^{i_{\ell}}\right)$ such that $R_{\ell}\left(d^{i_{\ell}}\right)<\rho$.

We refer to [9], in particular [9, Lemma 2.2], for more details.

\section{References}

1. Alarcón, S., Quaas, A.: Large number of fast decay ground states to Matukuma-type equations. J. Differ. Equ. 248, 866-892 (2010)

2. Battelli, F., Johnson, R.: On positive solutions of the scalar curvature equation when the curvature has variable sign. Nonlinear Anal. 47, 1029-1037 (2001)

3. Bianchi, G.: Non-existence and symmetry of solutions to the scalar curvature equation. Commun. Partial Differ. Equ. 21, 229-234 (1996) 
4. Bianchi, G.: The scalar curvature equation on $\mathbb{R}^{n}$ and $\mathbb{S}^{n}$. Adv. Differ. Equ. 1, 857-880 (1996)

5. Bianchi, G.: Non-existence of positive solutions to semilinear elliptic equations on $\mathbb{R}^{n}$ or $\mathbb{R}_{+}^{n}$ through the method of moving planes. Commun. Partial Differ. Equ. 22, 1671-1690 (1997)

6. Bianchi, G., Egnell, H.: An ODE approach to the equation $\Delta u+K u^{\frac{n+2}{n-2}}=0$, in $\mathbb{R}^{n}$. Math. Z. 210, 137-166 (1992)

7. Bianchi, G., Egnell, H.: A variational approach to the equation $\Delta u+K u^{\frac{n+2}{n-2}}=0$ in $\mathbb{R}^{n}$. Arch. Ration. Mech. Anal. 122, 159-182 (1993)

8. Cao, D., Noussair, E., Yan, S.: On the scalar curvature equation $-\Delta u=(1+\varepsilon K) u^{(N+2) /(N-2)}$ in $\mathbb{R}^{N}$. Calc. Var. Partial Differ. Equ. 15, 403-419 (2002)

9. Chen, C.C., Lin, C.S.: Blowing up with infinite energy of conformal metrics on $S^{n}$. Commun. Partial Differ. Equ. 24, 785-799 (1999)

10. Chen, C.C., Lin, C.S.: On the asymptotic symmetry of singular solutions of the scalar curvature equations. Math. Ann. 313, 229-245 (1999)

11. Cheng, K.S., Chern, J.L.: Existence of positive entire solutions of some semilinear elliptic equations. J. Differ. Equ. 98, 169-180 (1992)

12. Coddington, E., Levinson, N.: Theory of Ordinary Differential Equations. Mc Graw Hill, New York (1955)

13. Dalbono, F., Franca, M.: Nodal solutions for supercritical Laplace equations. Commun. Math. Phys. 347, 875-901 (2016)

14. Ding, W.Y., Ni, W.M.: On the elliptic equation $\Delta u+K u^{\frac{n+2}{n-2}}=0$ and related topics. Duke Math. J. 52, 485-506 (1985)

15. Flores, I., Franca, M.: Multiplicity results for the scalar curvature equation. J. Differ. Equ. 259, 4327-4355 (2015)

16. Franca, M.: Non-autonomous quasilinear elliptic equations and Ważewski's principle. Topol. Methods Nonlinear Anal. 23, 213-238 (2004)

17. Franca, M.: Structure theorems for positive radial solutions of the generalized scalar curvature equation. Funkc. Ekvacioj 52, 343-369 (2009)

18. Franca, M.: Fowler transformation and radial solutions for quasilinear elliptic equations. Part 2: nonlinearities of mixed type. Ann. Mat. Pura Appl. 189, 67-94 (2010)

19. Franca, M.: Radial ground states and singular ground states for a spatial-dependent $p$-Laplace equation. J. Differ. Equ. 248, 2629-2656 (2010)

20. Franca, M.: Positive solutions for semilinear elliptic equations: two simple models with several bifurcations. J. Dyn. Differ. Equ. 23, 573-611 (2011)

21. Franca, M.: Positive solutions of semilinear elliptic equations: a dynamical approach. Differ. Integr. Equ. 26, 505-554 (2013)

22. Franca, M., Johnson, R.: Ground states and singular ground states for quasilinear partial differential equations with critical exponent in the perturbative case. Adv. Nonlinear Stud. 4, 93-120 (2004)

23. Franca, M., Sfecci, A.: Entire solutions of superlinear problems with indefinite weights and Hardy potentials. J. Dyn. Differ. Equ. 30, 1081-1118 (2018)

24. García-Huidobro, M., Manasevich, R., Yarur, C.: On the structure of positive radial solutions to an equation containing $p$-Laplacian with weights. J. Differ. Equ. 223, 51-95 (2006)

25. Johnson, R., Pan, X.B., Yi, Y.F.: The Melnikov method and elliptic equation with critical exponent. Indiana Math. J. 43, 1045-1077 (1994)

26. Kawano, N., Ni, W.M., Yotsutani, S.: A generalized Pohozaev identity and its applications. J. Math. Soc. Jpn. 42, 541-564 (1990)

27. Kawano, N., Yanagida, E., Yotsutani, S.: Structure theorems for positive radial solutions to $\Delta u+$ $K(|x|) u^{p}=0$ in $\mathbb{R}^{n}$. Funkcial. Ekvac. 36, 557-579 (1993)

28. Li, Y., Ni, W.M.: On conformal scalar curvature equations in $\mathbb{R}^{n}$. Duke Math. J. 57, 895-924 (1988)

29. Lin, C.S., Lin, S.S.: Positive radial solutions for $\Delta u+K u^{\frac{n+2}{n-2}}=0$ in $\mathbb{R}^{n}$ and related topics. Appl. Anal. 38, 121-159 (1990)

30. Lin, L.S., Liu, Z.L.: Multi-bump solutions and multi-tower solutions for equations on $\mathbb{R}^{n}$. J. Funct. Anal. 257, 485-505 (2009)

31. Naito, Y.: Bounded solutions with prescribed numbers of zeros for the Emden-Fowler differential equation. Hiroshima Math. J. 24, 177-220 (1994)

32. Ni, W.M.: On the elliptic equation $\Delta u+K u^{\frac{n+2}{n-2}}=0$, its generalizations, and applications in geometry. Indiana Univ. Math. J. 31, 493-529 (1982)

33. Noussair, E.S., Yan, S.: The scalar curvature equation on $\mathbb{R}^{N}$. Nonlinear Anal. 45, 483-514 (2001) 
34. Papini, D., Zanolin, F.: On the periodic boundary value problem and chaotic-like dynamics for nonlinear Hill's equations. Adv. Nonlinear Stud. 4, 71-91 (2004)

35. Wei, J., Yan, S.: Infinitely many solutions for the prescribed scalar curvature problem on $\mathbb{S}^{N}$. J. Funct. Anal. 258, 3048-3081 (2010)

36. Yan, S.: Concentration of solutions for the scalar curvature equation on $\mathbb{R}^{N}$. J. Differ. Equ. 163, 239-264 (2000)

37. Yanagida, E., Yotsutani, S.: Classification of the structure of positive radial solutions to $\Delta u+K(|x|) u^{p}=$ 0 in $\mathbb{R}^{n}$. Arch. Ration. Mech. Anal. 124, 239-259 (1993)

38. Yanagida, E., Yotsutani, S.: Existence of nodal fast-decay solutions to $\Delta u+K(|x|)|u|^{p-1} u=0$ in $\mathbb{R}^{n}$. Nonlinear Anal. 22, 1005-1015 (1994)

39. Yanagida, E., Yotsutani, S.: Existence of positive radial solutions to $\Delta u+K(|x|) u^{p}=0$ in $\mathbb{R}^{n}$. J. Differ. Equ. 115, 477-502 (1995)

40. Yanagida, E., Yotsutani, S.: Global structure of positive solutions to equations of Matukuma type. Arch. Ration. Mech. Anal. 134, 199-226 (1996)

Publisher's Note Springer Nature remains neutral with regard to jurisdictional claims in published maps and institutional affiliations. 